
NIST Technical Note 1385

\section{Global Position System Receivers and Relativity}

Neil Ashby
Marc Weiss

Time and Frequency Division

Physics Laboratory

National Institute of Standards and Technology

325 Broadway

Boulder, Colorado 80303-3328

March 1999

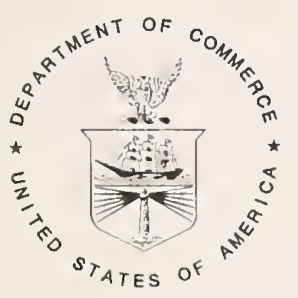

U.S. DEPARTMENT OF COMMERCE, William M. Daley, Secretary

TECHNOLOGY ADMINISTRATION, Gary R. Bachula, Acting Under Secretary for Technology

NATIONAL INSTITUTE OF STANDARDS AND TECHNOLOGY, Raymond G. Kammer, Director 
National Institute of Standards and Technology Technical Note Natl. Inst. Stand. Technol., Tech. Note 1385, 52 pages (March 1999) CODEN:NTNOEF

\section{U.S. GOVERNMENT PRINTING OFFICE \\ WASHINGTON: 1999}

For sale by the Superintendent of Documents, U.S. Government Printing Office, Washington, DC 20402-9325 


\section{Contents}

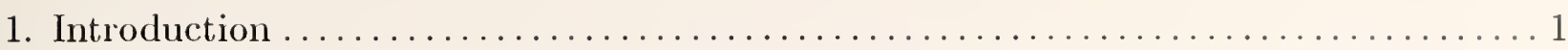

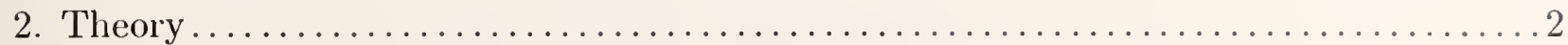

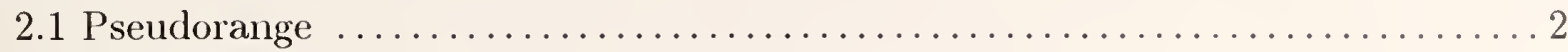

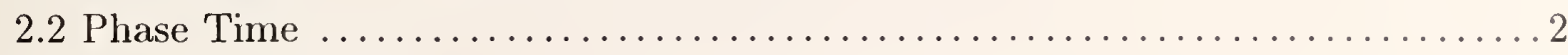

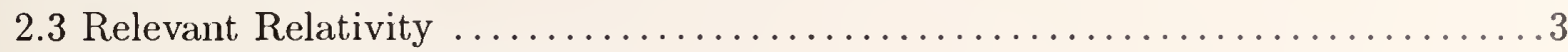

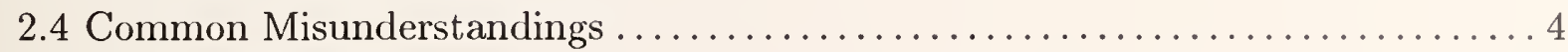

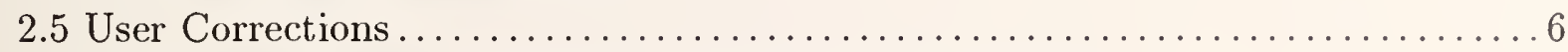

2.6 Relativistic Doppler Effect and Use of the GPS Carrier................. 7

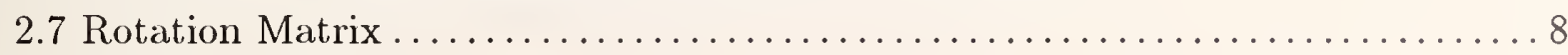

3. Time Tagging at Receiver ....................................... 9

3.1 Time Tagging at Receiver-General Prescription $\ldots \ldots \ldots \ldots \ldots \ldots \ldots \ldots$

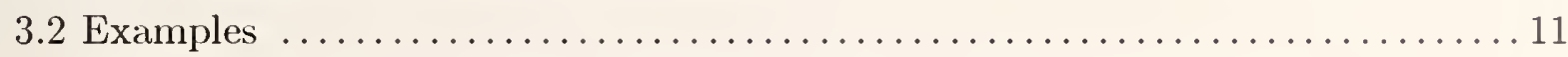

Example 3.21 Multi-Channel Receiver, Time Tagging at Receiver ......... 12

Example 3.211 Erroneous Use of ECEF Coordinates ................. 16

Example 3.212 Iteration With a Succession of Inertial Frames..............16

Example 3.213 The Benefit of Better Initialization................... 17

4. Time Tagging at Transmitter ................................. 18

4.1 Code Boundary Measurements ................................. 18

4.2 Phase Time................................................... 19

4.3 Accounting for Receiver Motion ............................... 20

4.4 Time Tagging at Transmitter-General Prescription ................... 22

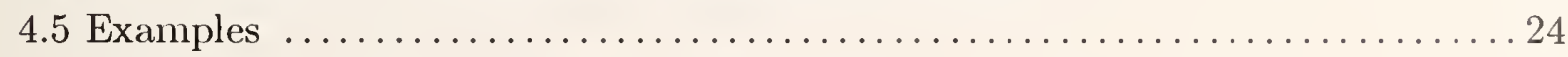

Example 4.51 Time Tagging at Transmitter ...................... 24

Example 4.52 Time Tagging at Transmitter, Neglecting Doppler Corrections 29

5. Time Transfer Receiver .......................................... 29

Example 5.1 Earth-Fixed Single Channel Timing Receiver .............. 32

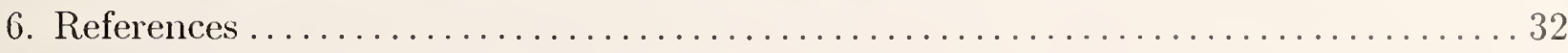

Appendix A. The Eccentricity Correction ............................ 35

Appendix B. Linearization Algorithm for Time Tagging at Multichannel Receiver .... 37

Appendix C. Linearization Algorithn for Tine Tagging at Transmitters ............ 39

Appendix D. Equivalence of Integrated Doppler and One-Way Light Time ........... 42

Appendix E. Numerical Check on First-Order Doppler Correction ................ 45 



\title{
GLOBAL POSITIONING SYSTEM RECEIVERS AND RELATIVITY
}

\author{
Marc Weiss \\ National Institute of Standards and Technology \\ Time and Frequency Division \\ 325 Broadway, Boulder, CO 80303-3324 \\ Neil Ashby \\ Department of Physics, Campus Box 390 \\ University of Colorado \\ Boulder, CO 80309-0390
}

\begin{abstract}
We illustrate the general methods for applying relativistic corrections needed by a GPS receiver in providing time or position to a user. We focus on estimating the geometric range delay, the time GPS signals require to propagate from the transmitter to the receiver in vacuum. We discuss several common uses of GPS including time tagging at the receiver. time tagging at the transmitter, and earth-fixed timing receivers. We include examples which illustrate these cases numerically.
\end{abstract}

\section{KEYWORDS}

GPS receivers; relativity; navigation; gravitational redshift: second-order Doppler shift; pseudorange.

\section{INTRODUCTION}

A GPS receiver must make two corrections that are related to relativity in order to provide time or position to a user. We discuss these corrections and focus mostly on estimating the geometric range delay $t_{D}$, the time for GPS signals to propagate from the transmitter to the receiver in vacuum. Proper estimation of $t_{D}$ is essential for solving for position or time. This is an application of the relativistic principle of the constancy of the speed of light, which states that electromagnetic signals travel in Euclidean straight lines with speed $c$ relative to an inertial reference franne. We present a few cases which apply to many common uses of GPS and illustrate them numerically.

We present the theory behind corrections with references given for any derivations not done here. Through the derivations, we show that the Interface Control Docunnent (ICDGPS-200) specifications, as issued by the Joint Progran Office of the Global Positioning 
System [1]. consistently cover the requirements of relativity down to within 1 ns or less. We show that the ICD specifications include relativity corrections with enough accuracy for most applications [2,3]. In particular, we discuss the relativistic Doppler effect, the formula for its instantaneous magnitude, and its relationship with typical GPS receiver operation. We also address the use of carrier-phase measurements, which is not discussed in the ICD.

Relativistic effects which are not usually modelled in GPS are: (a) a small effect on satellite clock rates due to the earth's oblateness and (b) a small time delay due to the slowing of electromagnetic signal propagation in the earth's gravitational field. The earth's oblateness contributes a very small constant rate correction for satellite clocks, which is so small (less than 50 ps per day) that it can be neglected. It also causes a periodic variation in the GPS satellite clock time having twice the orbital period and a peak-to-peak amplitude less than 200 ps. For a GPS satellite-to-user link the gravitational time delay is less than 200 ps [2].

Our goal is to give simple recipes, so that GPS users may understand how to implement receiver designs consistently with the requirements of relativity. We then back up these prescriptions with full explanations and derivations, so that those interested can understand where the prescriptions come from and what approximations are involved. The result is that this document provides a brief tutorial on how relativity is applied for users of the GPS broadcast ephemerides.

\section{THEORY}

\subsection{Pseudorange}

Generally, a GPS navigation receiver measures the arrival times, on a local clock, of signals from four or more different satellites. The receiver then uses these measurements along with information in the navigation messages to solve for four unknowns: user position $(x, y, z)$ and the receiver clock offset from GPS time. The signals from the satellites can be thought of effectively as continuous timing signals arriving at the receiver from the satellite clocks. The receiver has its own local clock for comparison. The user measures either (1) the times in the received timing signals at a specific local clock time, or (2) the arrival times on the local clock of a specific tine tag in the received timing signals. The difference between the reception time (according to the local clock) and the transmission time at one satellite (according to GPS time), multiplied by the speed of light, is called the pseudorange. These pscudoranges are used to solve for user position and tine.

\subsection{Phase Time}

In addition to times measured on real clocks, we can think of a particular phase point in the wave train between transmitter and receiver as mumerically equal to some time $\tau$, 
which follows the wave as it propagates. This "phase time" $\tau$ is transmitted from the satellite at SV clock time $t_{S V}$ and propagates to the receiver where it arrives at receiver clock time $t_{R}$. Thus the pseudorange is $c\left(t_{R}-t_{S V}\right)$.

\subsection{Relevant Relativity}

Several relativistic effects have already been incorporated into the GPS system so, for the ordinary user of broadcast ephemerides, only two relativistic corrections must be considered. First, the receiver must apply a correction to the transmitted time to account for relativistic effects arising from orbit eccentricity of the transmitting satellite. This is the $\Delta t_{r}$ term defined in the ICD. Second, the finite and universally constant speed $c$ of signals propagating in a vacuum from a transmitter to a receiver, relative to an inertial frame (the geometric path delay), must be accounted for. The receiver must also account for ionospheric and tropospheric delay corrections, which we do not consider here.

Three relativistic effects are germane to GPS. Rates of clocks in GPS are adjusted (as they are for International Atomic Time) to match the rate that clocks would run on the geoid of the earth. The geoid is a surface of constant gravitational potential in the rotating frame in which the effects (2) and (3) described below add to a constant value. The three relativity effects are as follows.

(1) Constancy of the speed of light and relativity of synchronization. GPS time is defined using the principle of the constancy of $c$ to synchronize an imagined system of clocks everywhere in space in the neighborhood of the earth (this is called Einstein synchronization). GPS satellite clocks are in principle adjusted to agree with this imagined system of clocks. This network of synchronized GPS clocks realizes a coordinate time, a system of self-consistent time markers with which to label events. This definition of GPS time requires a locally inertial coordinate system. GPS time is thus defined relative to an earth-centered inertial coordinate system (an ECIF), but the rate is set to match the rate at which clocks would run on the geoid. An ECIF is also used to simplify the paths of signals propagating from satellites, since, with sufficient accuracy for GPS, light travels in Euclidean straight lines at the speed $c$ in vacuun relative to such inertial franes [2].

(2) Second-order Doppler shift. A clock moving with respect to an ECIF rums slower relative to coordinate time in that ECIF than if it were at rest in the ECIF. This is the time dilation effect due to the magnitude of the relative velocity, sometines called the second-order Doppler effect. For satellites in GPS orbits, the fractional frequency offset needed to compensate for this is approximately $+8.3 \times 10^{-11}$ relative to the rate of clocks on the earth's geoid.

(3) Gravitational frequency shift. A clock at rest in a lower gravitational potential runs slower relative to coordinate time than if it were at rest in a ligher potential. This is called the gravitational red shift. Thus, standard clocks closer to the carth run slower than standard clocks farther away, since the gravitational potential becomes more negative 
closer to the earth. Clocks on GPS satellites run faster than clocks at rest on the earth's surface. Thus GPS satellite clock frequencies need to be adjusted by a fraction of about $-5.3 \times 10^{-10}$ relative to the earth's geoid, to compensate for this effect.

To compensate for the relativistic effects described in paragraphs (2) and (3) above for circular orbits, and as a consequence of the requirement that GPS satellite clocks run at the rate that a standard clock on the geoid would run, atomic clocks in GPS satellites are given a fixed fractional frequency offset of $-4.4645 \times 10^{-10}$. The user does not have to be concerned about these rate corrections.

These three relativistic effects, however, explain why the user must still apply two corrections. The first relativistic correction is the $\Delta t_{r}$ term defined in the ICD. This term corrects the satellite vehicle (SV) clock offset due to any eccentricity in the vehicle orbit. Eccentricity produces periodic time excursions from the nominal fixed fractional rate offset of $-4.4645 \times 10^{-10}$. The second correction applies to users' estimates of the geometric range delay, the delay from the transmitter to the receiver if the signal traveled in vacuum. Since an ECEF frame rotates relative to any inertial frame, light does not travel in a Euclidean straight line in an ECEF frame. It is incorrect to calculate the geometric range in an ECEF frame using the magnitude of the vector difference between the receiver's position at reception and the transmitter position at transmission. The distance is most easily calculated in an ECIF where signals do travel in Euclidean straight lines at the speed of light, the constant $c$.

Many ECI coordinate systems, differing by constant spatial rotations from each other, may serve these purposes. All frames that have the same origin at the earth's center and do not rotate with respect to the "fixed" stars will define simultaneity in the same way. All such frames are equivalent for determining the propagation delay. Yet users usually need to refer their positions to the earth. For example they may need their coordinates in the WGS-84 coordinate system, the ECEF frame upon which the broadcast ephemerides are based. Users fixed on the earth who want time from GPS often know their coordinates as constants in the ECEF frame. In fact, most users would like to know their coordinates in the ECEF frame.

\subsection{Common Misunderstandings}

There has been confusion in the GPS community over a number of issues related to relativity $[5,6]$. For example, arguments have been presented that we need to define a coordinate system in the rest frame of the receiver or perhaps in that of the transmitter. Since measurements are made in the receiver, it might at first appear necessary to use a coordinate system comoving with the receiver. However, such arguments overlook the fact that relativistic effects -indeed, any physical effects-can be described in any coordinate system.

Another argunent that has been advanced is that we nced to compute corrections in 
the rotating coordinate system of the ECEF frame, since that is the coordinate system in which the user ultimately wishes to find his/her position. The relativity of simultaneity has sometimes been presented as a principle necessitating such constructions.

Although measurements are made with a particular instrument and an instantaneous rest frame is associated with that instrument, it is not necessary to use that rest frame to analyze the data. This is a common misunderstanding underlying many of the errors in the literature: the belief that we must always use reference frames which are comoving with the transmitter or receiver. The user does not need a coordinate system whose origin coincides with the user's trajectory in spacetime to analyze effects local to the user.

It is a common practice in physics to choose a convenient coordinate system to solve a problem. This is particularly germane to relativity, where the principle of covariance states that, if a problem is understood in one coordinate system, it can be understood in any other by making appropriate transformations. Thus, even though neither the user nor the transmitter are at rest in an ECIF, the user may choose an ECIF to simplify computations of position, then transform those coordinates to the ECEF frame, the WGS-84 system. The pseudorange that the user measures is the same regardless of which coordinate system the user chooses. It is simpler to use an ECIF for computations than to try to make all corrections in the rotating ECEF coordinate system.

The fundamental measurement of a GPS receiver is the pseudorange. This is a measurement of the difference between the time associated with the phase of a GPS signal and the time on the local clock at the arrival of that phase. We will discuss why this measurement has the same value regardless of which coordinate system the user chooses to do calculations.

The phase of an electromagnetic wave is a relativistic scalar. This follows simply because the phase is the relativistic scalar product of two fourvectors: the wave propagation fourvector and the position fourvector. This means the phase has the same numerical value in all reference frames. It does not matter whether this phase is evaluated in the earthcentered inertial frame, the rest frame of the transmitter, or the rest frame of the receiver.

For GPS signals, GPS time is encoded in the transmitted pseudorandom noise (PRN) signal. The bits in the PRN code are bi-phase modulated on the L-band carrier. Thus when the time arrives for new bit of the code, the phase of the carrier either reverses $180^{\circ}$ or it does not, corresponding to whether the next bit is one or zero. The receiver measures the time of arrival of a GPS signal by comparing it with a locally generated replica of that signal. The receiver correlates the received signal against the local replica and examines the correlator output to find the correlation maxinnm, then locks the local code replica to the transmitted code. Maintaining this lock in general requires adjusting the frequency and time offset of the code replica in the correlator relative to the local clock. The receiver system then determines the tine difference between the received platise tine and the tinne on the local clock. It is as if the wave front of the GPS signal hais an mannbiguous tine 
marker on it. Thus. the user can measure the phase of the received signal, hence the time as received, against the time of the receiver's local oscillator. The difference between the phase time as received and the time of the receiver's local oscillator, after multiplication by $c$ to give a quantity with units of length, is the pseudorange.

We can see why the pseudorange is independent of which coordinate system the receiver uses to solve for position and/or time. This follows since the phase is a relativistic scalar and the measurement is made when the receiver clock is co-located with that GPS signal phase. The measurement is essentially the time difference between the local clock time at a particular point on that clock's space-time path, and the GPS signal phase time at the same spacetime point.

The navigation problem could be completely solved using only the ECEF frame. It is complicated in the ECEF frame because light and hence GPS signals no longer travel in Euclidean straight lines. The time for a signal to travel from transmitter at $\mathbf{R}$ to receiver at $\mathbf{r}$ is not $|\mathbf{r}-\mathbf{R}| / c$. Similarly we could set up a coordinate system in either the rest frame of the receiver or of the transmitter. However, we emphasize that the problem is greatly simplified by analyzing the problem in an ECIF, then transforming results appropriately to the ECEF frame. This is simplified by the fact that the time coordinate in ECIF's as we have defined them is identical to that in the ECEF frame. Thus, for example, the relativity of simultaneity is not an issue because time, and simultaneity, are defined the same way in all these coordinate systems.

\subsection{User Corrections}

At any arbitrarily chosen instant the ECEF frame coincides with an ECI frame having identical $x, y, z$ axes, but not rotating. Removing the rotation of the earth from the ECEF frame defines a coordinate system that is close enough to an inertial frame to serve for estimating the path delay. As time passes, the ECEF system rotates while this ECI system remains behind. so to speak. Any such fixed coordinate system may serve as an ECIF for estimating geometric range, using the Euclidean distance between the coordinates in the ECIF of the satellite at transmission and the coordinates of the receiver at reception. The time for the signal to travel this path, the geometric path delay $t_{D}$, may be estimated to high accuracy as the geometric range divided by the defined speed of light $c$. The slowing of electronagnetic signal speed in earth's gravitational field (the Shapiro time delay) amounts to less than 200 ps delay for GPS users near earth. [2]

The principle of the constancy of the speed of light in an inertial frame requires that an ECIF be used for geometric path delay if it is calculated by dividing the Euchidean distance by c. Using such an ECIF greatly simplifies the problem of solving for a GPS user's position or time. Whereas GPS is intended to provide users with their position or time in the ECEF system, if we use ECEF coordinates for geometric range with the simple Euclidean distance metric and divide by $c$ to obtain geometric path delay, we would 
make significant errors since we would be ignoring motion of the receiver during the signal propagation time.

In general, a navigation solution requires pseudorange measurements from at least four satellites. These are used to obtain geometric range delay estimates, which in turn are used to solve for position. To use the simplicity of the Euclidean distance metric that comes with an ECIF, all satellite positions at the transmission epochs must be transformed into the common ECIF. The user may then solve for the receiver position in this coordinate frame, and for the GPS time $t$ corresponding to this solution. Finally, the user must find the receiver coordinates in the ECEF frame at the GPS time $t$ by accounting for the rotation of the ECEF frame between the chosen moment $t_{C}$ at which an ECI frame is defined, and the GPS time $t$.

With a receiver using four satellites we generally have five different times of interest as natural candidates for $t_{C}$ : either a single GPS transmission time and four different reception times (time tagging at transmission time), or a single reception time and four different transmission times (time tagging at reception time). In any case an ECI frame can always be found which coincides with the ECEF frame at a chosen instant of time, and in this sense the ECEF frame can determine an inertial coordinate system. This answers the need for a coordinate systen in which light travels in straight lines with speed $c$ during the transmission of the signals, and in which all the GPS satellite clocks can in principle be synchronized. We will give a number of examples below for using this general prescription. Our focus here will remain on relativistic corrections for GPS users. We do not consider corrections for nonrelativistic delays: ionospheric plasma delays, tropospheric delays due to water vapor, multipath interference, or receiver system delays. Nonrelativistic delays must certainly be accounted for; however, here our topic is relativity.

\subsection{Relativistic Doppler Effect and Use of the GPS Carrier}

Many receivers that make use of the GPS carrier use only the accumulated phase to smooth code measurements. In this case the instantaneous relativistic Doppler shift need not be considered. As we show in Appendix D, the integrated carrier as received over one cycle of the code is equivalent to the change in geometric range over that interval. Thus if a receiver using accumulated Doppler shifts kecps track of geometric range. then it automatically keeps track of the relativistic Doppler shift. Nevertheless some receivers may use the instantaneous frequency of the received signals for some purpose. Such a receiver must account for the relativistic Doppler effect.

A receiver system that uses the instantaneous Doppler slift of the received carricr signal must also correct for the frequency slift of the received signal within the framework of relativity. As the true range between the satellite and receiver clanges due to relative motion, the carrier frequency changes due to the Doppler effect. The relationship between the received frequency $f$, and the transmitted frequency. or proper frequency $F$, is derived 
in Appendix D with accuracy sufficient for GPS. This relationship is $[2,3,4]$

$$
\frac{f\left(1+\Delta \Phi\left(\mathbf{r}_{R}\right) / c^{2}-\mathbf{v}^{2} / 2 c^{2}\right)}{1-\frac{N \cdot \mathbf{v}}{c}}=\frac{F\left(1+\Delta \Phi\left(\mathbf{r}_{T}\right) / c^{2}-\mathbf{V}^{2} / 2 c^{2}\right)}{1-\frac{\mathbf{N} \cdot \mathbf{V}}{c}}
$$

where

$$
\begin{aligned}
\mathrm{V}= & \text { transmitter velocity in ECI coordinates } \\
\mathbf{v}= & \text { receiver velocity in ECI coordinates } \\
\mathrm{N}= & \text { ECI unit vector in the direction of signal propagation, } \\
\Delta \Phi= & \text { gravitational potential difference between } \\
& \quad \text { a clock and a reference clock on the geoid. }
\end{aligned}
$$

If gravitational potential differences can be ignored. the relationship between received and transmitted frequency can be written without approximation as

$$
\frac{f}{\gamma(\mathbf{v})\left(1-\frac{\mathbf{N} \cdot \mathbf{v}}{c}\right)}=\frac{F}{\gamma(\mathbf{V})\left(1-\frac{\mathbf{N} \cdot \mathbf{V}}{c}\right)}
$$

where

$$
\gamma(v)=\frac{1}{\sqrt{1-\frac{v^{2}}{c^{2}}}} .
$$

The change in frequency is closely related to the rate of change of range. In fact, in Appendix D we show that the relativistic Doppler frequency shift equation can be derived by differentiating the geometric path delay $[4,6]$. In Appendix D we have included for completeness the effect of the gravitational frequency shift to order $c^{-2}$, which is sufficient for users of the GPS. This proves the conceptual equivalence, within the framework of Special and General Relativity. of the methods of pseudorange (to obtain an instantaneous position solution) and integrated Doppler frequency (to obtain changes in position). A user of integrated Doppler frequency accounts for changes in the geometric path delay simply by integrating the relativistic Doppler shift and relating this observable quantity to the change in geometric range. This is discussed in detail in Appendix D.

\subsection{The Rotation Matrix}

Almost always the user of the broadcast ephemerides will want to use coordinate transformations which correspond to rotations of the coordinate system about the WGS$84 z$ axis. For convenience we write out here an example of such a rotation matrix. Consider an ECIF with $z$ axis which coincides with the defined WGS-84 axis. Let the position coordinates of some point of interest in these ECI coordinates be denoted by

$$
X_{E C I}=\left[\begin{array}{l}
x_{E C I} \\
y_{E C I} \\
z_{E C I}
\end{array}\right]
$$


Suppose that the ECI axes coincide with the ECEF axes at the time $t_{C}$. To calculate the ECEF coordinates of the point at time $t$, note that the angle through which the ECEF coordinates have rotated is $\Theta(t)=\dot{\Omega}_{e}\left(t-t_{C}\right)$, where $\dot{\Omega}_{e}=7.2921151467 \times 10^{-5} \mathrm{rad} / \mathrm{s}$ is the WGS-84 value of the earth rotation rate. Then the coordinates of the point in the ECEF frame are given by

$$
X_{E C E F}=R^{S}(t) X_{E C I}=\left[\begin{array}{ccc}
\cos \Theta(t) & \sin \Theta(t) & 0 \\
-\sin \Theta(t) & \cos \Theta(t) & 0 \\
0 & 0 & 1
\end{array}\right]\left[\begin{array}{c}
x_{E C I} \\
y_{E C I} \\
z_{E C I}
\end{array}\right] .
$$

This is sometimes called a "passive" rotation - that is, a rotation of the coordinate axes keeping physical position vectors unchanged. In eq (4) the time interval $t-t_{C}$ must be small since the earth rotation rate actually varies. With $t-t_{C}$ as large as $3 \mathrm{~s}$, errors no larger than $0.05 \mathrm{~mm}$ are introduced.

\section{TIME TAGGING AT THE RECEIVER}

Generally, a GPS navigation user measures the arrival times of signals from four different satellites and uses them to solve for four unknowns: position $x, y, z$ and time $t$. A common way of doing so is to make the four measurements at one instant at the receiver ("time tagging at the receiver"). Another method is to use signals which left the satellites at a common GPS time ("time tagging at the transmitters"). The latter method is more complex, both because the signals are not received simultaneously, and because GPS times on the satellite clocks must be obtained by applying transmitted clock correction coefficients. That is, the clocks on the satellites are synchronized only if the user applies transmitted clock corrections. The GPS satellite clocks themselves may differ from GPS system time by up to $1 \mathrm{~ms}$ in the underlying ECI reference frame [1, Sections 20.3.3.3.1.9-20.3.3.3.3.1]. Thus in the case of time tagging at the transmitters we must account both for the motion of the user during the intervals between transmission and reception, and the differences between SV clock time and GPS system time. On the other hand, as we shall show, time tagging at the transmitter can be used for navigation while avoiding most rotations.

\subsection{Time Tagging at the Receiver-General Prescription}

(1) At a chosen reception time, measure the transmission times using the received pseudo-random noise (PRN) codes from four satellites. This gives us the tines $t_{S V}$ according to the SV clocks at transunission for the signals received at the chosen reception time.

We need the GPS time of transmission for each satellite and the common reception time according to the (possibly biased) local clock. We obtain the time of reception from on local clock. The transunssion 
time is encoded in the received signal. Since we are locked to the code, we can determine the offset of the locally generated PRN sequence required to maintain lock.

(2) Apply the prescription of Table 20-IV of the ICD-200, for corrections to obtain GPS system time $t$, at transmission.

This includes the eccentricity correction $\Delta t_{r}$, described in the ICD200 , section 20.3.3.3.3.1, and is an effect due to relativity. $\Delta t_{r}$ is a correction which applies to the SV clock; it is the same correction no matter where the receiver is or how the receiver is moving. The value of $\Delta t_{r}$ will generally be different for the clocks in different satellites. For example, for a clock in a satellite of orbital eccentricity $e$, transmitting at an instant when the eccentric anomaly is $E$, the correction to be subtracted from the transmitted time is

$$
\Delta t_{r}=F \times 10^{-10} e \sqrt{A} \sin E,
$$

where

$$
F=-4.442807633 \mathrm{~s} / \mathrm{m}^{1 / 2},
$$

$A$ is the semi-major axis of the satellite in meters, $e$ is the orbital eccentricity, and $E$ is the eccentric anomaly. Values of these quantities are transmitted in the navigation message.

(3) Compute each satellite's position in the ECEF frame at its transmission time.

This is a straightforward application of the equations of Table 20-IV of the ICD-200 [1] for determining the $x, y, z$ coordinates of the satellite at the instant of transmission. Note that the broadcast message gives the satellite positions at the instant of tran'smission in ECEF coordinatesspecifically in the WGS-84 reference frame.

(4) Choose an ECIF for computation of the path delays.

This choice is arbitrary, but some choices are more convenient than others. Simplification may sometimes occur if the choice is appropriately made. A natural choice for the case of time tagging at the receiver is to freeze the ECEF frame at a time close to the expected GPS time of reception.

(5) Transform the ECEF coordinates of each SV obtained in step (3) into the chosen ECIF.

This will normally require at least three, and perhaps four, of such ECEF position vectors to be rotated. If we freeze the ECEF frame at the instant of transmission of one of the satellites, only three of these 
rotations will be needed. Alternatively, we nay frecze the ECEF frame at any instant to define an earth-centered inertial system. If we choose the GPS time equal to the local clock's reception time, the later corrections may be small. If the local clock is sufficiently close to GPS time at the instant of reception, then the last step below, step (7), would not need to be done.

(6) Solve the path delay equations for the receiver's position and time.

This can be done by linearizing the propagation delay equations and solving them iteratively. Here we solve simultaneously for geometric range delay $t_{D}$ and user position. The user's motion through the ECIF during propagation is included in these $t_{D}$ estimates. Note that other contributions to path delay which we do not consider here, such as ionospheric and tropospheric delays, should be incorporated in this process. We use an initial estimate of position to solve for the linearized corrections to receiver position and the time offset. If these corrections are not small enough for the user, they may be used to obtain a next estimate of position and time. This, in turn, may be used to obtain the next linearized correction for position and time offset. This iterative process converges rapidly.

(7) Rotate the user's position coordinates into the ECEF reference frame.

After finding the user's position in the chosen ECI coordinate system and the correct GPS systcm time, the receiver's ECI position coordinates are rotated into the ECEF reference frame at the instant corresponding to the measured reception time. If the ECI frame chosen in step (4) is sufficiently close to the ECEF frame at the instant of reception, this step may be omitted.

\subsection{EXAMPLES}

In these examples we use orbital data representing the GPS constellation as it approximately was in March of 1995. Values of the semi-major axes, eccentricitics, and inclinations have been randomized within very small, but reasonable limits. Keplerian orbit algorithms are used to propagate the SV orbits forward in time and to accurately compute SV eccentric anomalies. Values of $\Delta t_{S V j}$ are randomized using a uniform distribution within a $1 \mathrm{~ms}$ range. The SV identification numbers used are fictitious. However. the actual transmitter and receiver data used in these exanples are selected from annong many possibilities resulting from a sinulation of the entire GPS constellation. The data are thus obtained from a simulation where "truth" is accurately known and can be compared with navigation solutions. 


\section{Example 3.21 Multi-Chamel Receiver, Time Tagging at the Receiver}

We choose four satellites from this constellation in view from our receiver location with elevations above the horizon greater than $20^{\circ}$. Suppose the receiver is truly at geocentric ECEF latitude and longitude $35^{\circ} \mathrm{N}, 0^{\circ} \mathrm{E}$, and distance $R=6378136.3 \mathrm{~m}$ from earth's center. Suppose the user wants to make a measurement of position and GPS time at his/her clock time $t=37239.9994223656 \mathrm{~s}$ of the week, and that this just happens to be GPS time $t=37240$ seconds exactly, for purposes of illustration.

The actual receiver ECEF coordinates at this instant, which we give here for comparison with the navigation solution, will be

$$
\mathbf{X}_{W G S 84}=\left[\begin{array}{c}
R \cos 35^{\circ} \\
0 \\
R \sin 35^{\circ}
\end{array}\right]=\left[\begin{array}{c}
5224663.389 \mathrm{~m} \\
0 \\
3658348.690 \mathrm{~m}
\end{array}\right] .
$$

Such accuracy is not justified, but we are specifying positions within $1 \mathrm{~mm}$ so that the convergence of the algorithms can be checked.

We follow the step numbering from the prescription given above.

(1) Suppose we already have the measured SV clock times $t_{S V i}$ for the signals received at the chosen reception time.

The reception time is specified on the local clock, which is $t=$ $37239.9994223656 \mathrm{~s}$. This just happens to be at GPS coordinate time $t=37240.0000000000 \mathrm{~s}$ exactly and corresponds to a pseudorange $c \times 0.075 \mathrm{~ms}$ for SV 1 . The GPS time should be recovered during the computations.

(2) We apply the prescription according to Sect. 20.3.3.3.3.1 from the ICDGPS-200 describing the user algorithm. We then obtain the system time $t_{i}$, the GPS time of transmission, for each satellite $i$. The relativistic eccentricity correction is part of this calculation. (The subscript $i$ is not used in the ICD, but is added here for clarity.) The data we actually use in the present example were generated from a computer simulation which propagated orbits and clocks for the entire GPS constellation forward in time. In this simulation the offsets $\Delta t_{S V i}$ were randomly generated.

(3) Using the broadcast ephemeris, we obtain the ECEF coordinates of the satellites from the prescriptions given in Table 20-IV of the ICD-GPS-200. From the GPS time $t_{i}$, the time interval $t_{k}=t_{i}-t_{o e}$ from ephemeris reference epoch is calculated. Then $t_{k}$ may then be used in the algorithm for computation of ephemerides. This gives the $x, y, z$ coordinates of the satellite, in the ECEF frame, at its transmission epoch. Table 1 gives the results after these steps. (These data are actually generated from a computer simulation of the GPS constellation.) 
Table 1. GPS satellite positions in ECEF coordinates.

\begin{tabular}{ccccc}
\hline SV\# & Transmission epoch $t_{i}(\mathrm{~s})$ & $x_{i}(\mathrm{~m})$ & $y_{i}(\mathrm{~m})$ & \multicolumn{1}{c}{$z_{i}(\mathrm{~m})$} \\
\hline 1 & 37239.9244223656 & 13005878.255 & 18996947.213 & 13246718.721 \\
2 & 37239.9207133918 & 20451225.952 & 16359086.310 & -4436309.875 \\
3 & 37239.9253078700 & 20983704.633 & 15906974.416 & 3486595.546 \\
4 & 37239.9293463539 & 13798849.321 & -8706113.822 & 20959777.407 \\
\hline
\end{tabular}

In Table 1, GPS system times $t_{i}$ rather than the time intervals $t_{k}=t_{i}-t_{o e}$ from ephemeris reference epoch, are used to label the events. The subscript $i$ varying from 1 to 4 labels data from the different satellites. Table 1 assumes that the eccentricity correction discussed in Appendix A has already been applied to obtain the transmission epoch data given in the second column of the table. The position coordinates of each satellite are given in the ECEF frame, which is turning in inertial space.

(4) To rotate from ECEF to ECIF coordinates, the inverse of the rotation matrix of eq (4) is required. Thus we need $\left(R^{S}\left(t_{i}-t_{C}\right)\right)^{-1}$, which must be calculated and applied to the coordinates of each satellite individually. That is, $t_{i}$ will be different for each satellite, but $t_{C}$ is the same for all satellites. For this example, we choose an ECIF by fixing the rotation of the ECEF frame at $t_{C}=37239.0000000000 \mathrm{~s}$. We stress that this choice is arbitrary-better choices are usually available. The purpose of this choice is to illustrate its arbitrariness and also to illustrate how rapidly the iteration algorithm converges. With this choice, for example, the rotation matrix for satellite 3 is

$$
\left(R^{S}\left(t_{3}-t_{C}\right)\right)^{-1}=\left[\begin{array}{ccc}
\cos \dot{\Omega}_{e}\left(t_{3}-t_{C}\right) & -\sin \dot{\Omega}_{e}\left(t_{3}-t_{C}\right) & 0 \\
\sin \dot{\Omega}_{e}\left(t_{3}-t_{C}\right) & \cos \dot{\Omega}_{e}\left(t_{3}-t_{C}\right) & 0 \\
0 & 0 & 1
\end{array}\right]
$$

and $\dot{\Omega}_{e}\left(t_{3}-t_{C}\right)=0.9253078700 \cdot \dot{\Omega}_{e}=6.747451534 \times 10^{-5}$. Corresponding transformations are applied to each of the transmitted data sets.

(5) We transform the coordinates in Table 1 into this ECIF. The rotation matrix is different for each transmission epoch. We use a subscript. $C$ to indicate that the inertial system has been arbitrarily chosen. The results from transforming to the ECI frame are given in Table 2.

(6) The propagation delay equations to be solved are discussed in Appendix B. We give a quick summary here. The notation is as follows. The four GPS satellites, at GPS tines $t_{1}, t_{2}, t_{3}, t_{4}$ send ont signals from the ECIF locations 
Table 2. Transmitted data transformed to the chosen ECI system

\begin{tabular}{ccccc}
\hline SV \# & Transmission Epoch $t_{i}^{\prime}(\mathrm{s})$ & $x_{C i}(\mathrm{~m})$ & $y_{C i}(\mathrm{~m})$ & \multicolumn{1}{c}{$z_{C i}(\mathrm{~m})$} \\
\hline 1 & 37239.9244223656 & 13004597.642 & 18997823.895 & 13246718.721 \\
2 & 37239.9207133918 & 20450127.566 & 16360459.358 & -4436309.875 \\
3 & 37239.9253078700 & 20982631.270 & 15908390.245 & 3486595.546 \\
4 & 37239.9293463539 & 13799439.294 & -8705178.668 & 20959777.407 \\
\hline
\end{tabular}

$\mathbf{r}_{1}, \mathbf{r}_{2}, \mathbf{r}_{3}, \mathbf{r}_{4}$, respectively. (The subscripts now label data from the different satellites, after transforming to the chosen ECI reference frame. The rotations do not affect the times.) These four signals are received simultaneously at time $t$ by a receiver at position $\mathbf{r}_{C}$. The problem is to determine $t$ and $\mathbf{r}_{C}$ at the receiver. The velocity of the receiver does not have to be considered. The receiver's position $\mathbf{r}_{C}$ at the time of reception $t$ will be determined by the solution of four equations which express the condition that the speed of propagation is $c$. The four equations to be solved are

$$
\left(\mathbf{r}_{C}-\mathbf{r}_{j}\right)^{2}-c^{2}\left(t-t_{j}\right)^{2}=0 ; \quad j=1,2,3,4
$$

However, if the position $\mathbf{r}_{C}$ and time $t$ are known approximately, the equations can be reduced to a system of four linear equations which can be solved by standard matrix inversion techniques. These equations are derived in Appendix B, and are

$$
\left(\mathbf{r}^{(i)}-\mathbf{r}_{j}\right) \cdot \Delta \mathbf{r}-c^{2}\left(t^{(i)}-t_{j}\right) \Delta t=\frac{1}{2}\left(c^{2}\left(t^{(i)}-t_{j}\right)^{2}-\left(\mathbf{r}^{(i)}-\mathbf{r}_{j}\right)^{2}\right) .
$$

In eq (10), the quantity $c\left(t^{(i)}-t_{j}\right)$ is the $i$ th estimate of the pseudorange from the receiver to the $j$ th satellite, and $\mathbf{r}_{C}^{(i)}$ is the $i$ th estimate of the receiver position in ECI coordinates. Equation (10) is a system of linear inlomogeneous equations in the corrections $\Delta \mathbf{r}, \Delta t$, which can be solved by matrix inversion. The matrix of coefficients of the unknowns $\Delta \mathbf{r}$ and $\Delta t$ will usually be nonsingular, unless the configuration of satellites is so unfavorable that eqs (9) do not have a solution (such a condition is possible.) The solutions obtained will be approximate, but can be used to obtain new trial values; the iteration can be repeated as many times as necessary to obtain the accuracy required.

To illustrate this process in the present case, as our initial guess at receiver position we take a worst case and assume the receiver is at the center of the earth. Also, we do not know the receiver clock bias. In this exannple we assume the clock bias is such that the reception event 
occurs at $t=t_{1}+0.07500 \mathrm{~s}$. Then Table 3 gives the results at each stage of the iteration.

The calculation converges to within $1 \mathrm{~m}$ after three iterations. The time at reception on the receiver clock, determined by the solution, is

$$
t_{R}=37240.0000000000 \mathrm{~s} .
$$

(7) These results must lastly be transformed into the WGS84 system by applying the rotation $R^{S}\left(t_{R}-t_{C}\right)$. That is, to obtain WGS84 coordinates at the instant of reception, we must use a sidereal rotation corresponding to that instant. The necessary rotation matrix is

$$
R^{S}\left(t_{R}-t_{C}\right)=\left[\begin{array}{ccc}
\cos \dot{\Omega}_{e}\left(t_{R}-t_{C}\right) & \sin \dot{\Omega}_{e}\left(t_{R}-t_{C}\right) & 0 \\
-\sin \dot{\Omega}_{e}\left(t_{R}-t_{C}\right) & \cos \dot{\Omega}_{e}\left(t_{R}-t_{C}\right) & 0 \\
0 & 0 & 1
\end{array}\right]
$$

and $\dot{\Omega}_{e}\left(t_{R}-t_{C}\right)=7.2921151467 \times 10^{-5}$ because in this case, $t_{R}-t_{C}$ happens to be exactly $1 \mathrm{~s}$. Upon applying this rotation to the position coordinates given in the last line of Table 3 , the measured values of the receiver position are

$$
\mathbf{X}_{W G S 84}=\left[\begin{array}{c}
5224663.388 \mathrm{~m} \\
0.000 \mathrm{~m} \\
3658348.689 \mathrm{ml}
\end{array}\right]
$$

These agree with the true position coordinates to within a millimeter.

Table 3. Results of iterative solution of propagation delay equations.

\begin{tabular}{ccccc}
\hline Trial \# & User's x position & User's y position & User's z position & User's clock time \\
\hline 0 (start) & $0 \mathrm{~m}$ & $0 \mathrm{~m}$ & $0 \mathrm{~m}$ & $37239.9994223656 \mathrm{~s}$ \\
1 & $5057363.392 \mathrm{~m}$ & $2355.126 \mathrm{~m}$ & $3541092.792 \mathrm{~m}$ & $37239.9975323058 \mathrm{~s}$ \\
2 & $5226931.552 \mathrm{~m}$ & $354.224 \mathrm{~m}$ & $3659938.391 \mathrm{~m}$ & $37240.0000334559 \mathrm{~s}$ \\
3 & $5224663.780 \mathrm{~m}$ & $380.983 \mathrm{~m}$ & $3658348.973 \mathrm{~m}$ & $37240.0000000060 \mathrm{~s}$ \\
4 & $5224663.374 \mathrm{~m}$ & $380.988 \mathrm{~m}$ & $3658348.689 \mathrm{~m}$ & $37240.0000000000 \mathrm{~s}$ \\
5 & $5224663.374 \mathrm{~m}$ & $380.988 \mathrm{~m}$ & $3658348.689 \mathrm{~m}$ & $37240.0000000000 \mathrm{~s}$ \\
\hline
\end{tabular}


Table 4. Iterative solution ignoring earth rotation.

\begin{tabular}{ccccc}
\hline Trial \# & User's x position (m) & User's y position $(\mathrm{m})$ & User's z position (m) & User's clock time (s) \\
\hline 0 (start) & 0 & 0 & 0 & 37239.9994223656 \\
1 & 5057359.485 & 2012.579 & $3541090.386 \mathrm{~m}$ & 37239.9975323230 \\
2 & 5226927.086 & 00.194 & $3659935.704 \mathrm{~m}$ & 37240.0000334693 \\
3 & 5224659.325 & 27.107 & $3658346.291 \mathrm{~m}$ & 37240.0000000196 \\
4 & 5224658.919 & 27.112 & $3658346.008 \mathrm{~m}$ & 37240.0000000136 \\
5 & 5224658.919 & 27.112 & $3658346.008 \mathrm{~m}$ & 37240.0000000136 \\
\hline
\end{tabular}

\section{Example 3.211 Erroneous Use of ECEF Coordinates}

One might think that effects due to earth rotation, during the very small signal propagation times from transmitters to receiver, were sufficiently small that transforming to ECI coordinates was not necessary. Let us therefore test this with the example data given in Table 1. We again initialize the iteration as in Example 3.21, above. The results of the iteration, using the same algorithm as before are given in Table 4.

In this case, the solutions are in error by a significant amount. The receiver time is off by $14 \mathrm{~ns}$ and the position is in error by almost $30 \mathrm{~m}$. Thus, it is an error to ignore earth rotation during the propagation of signals from transmitters to receiver.

However, these results suggest an approach which could result in a computationally efficient strategy for the receiver. Suppose that the receiver is continually monitoring signals from four or more satellites and keeping track of its approximate position by neglecting earth rotation, as in Example 3.211 above, but that an accurate position is not needed until some particular moment. At that moment, the erroneous position and time could be used as a starting point for a correct iterative solution of the one-way light time equations. Since the starting point is not far off, this iteration should converge very rapidly. Thus, transformations to the chosen inertial frame $C$ would be made only when an accurate position and time were needed, eliminating much computation at other times.

\section{Example 3.212 Iteration with a Succession of Inertial Frames}

Another approach is to consider a succession of inertial frames in the iteration process, each of which is aligned with the WGS84 frame at the assumed reception time. This would have the nice advantage that at the end of the iteration, the ECIF would be exactly aligned with the WGS84 frame, so no final rotation would be necessary. The disadvantage is that the ECIF coordinates of the transmission events must be recomputed at each stage of the iteration. Thus in the internediate stages of iteration more computation is required. During actual use, we might have very good estimates of the receiver position available to start the iteration. Thus, very few iterations could yield receiver position to sufficient 
accuracy, making this approach favorable.

To illustrate this approach, we shall use a succession of equations of the form

$$
\mathbf{X}_{C}=R^{C}\left(t_{C}\right)\left(R^{S}\right)^{-1} \mathbf{X}_{W G S 84}=\left(R^{S}\left(t-t_{C}\right)\right)^{-1} \mathbf{X}_{W G S 84}
$$

to obtain inertial coordinates of the transmission events. We will first make a reasoned guess for the receiver time $t_{R}$ and then set $t_{C}=t_{R}$. One iteration will yield a more accurate estimate for $t_{R}$. We will then choose $t_{C}$ to be this new value and recompute satellite transmission event data for this new $t_{C}$, and repeat the process as often as necessary. The results are given in Table 5 .

\section{Example 3.213 The Benefit of Better Initialization}

It pays to be more careful with our initial guesses. To illustrate this, let us take as our initial time the latest transmission epoch plus $75 \mathrm{~ms}$ and take the initial position to be the subsatellite point of the latest transmission epoch. Again using a succession of inertial frames as in the above example, the results are given in Table 6 . What has been achieved is more rapid convergence, compared with the previous example.

Table 5. Iterative solution with a succession of inertial frames.

\begin{tabular}{ccccc}
\hline Trial \# & User's x position $(\mathrm{m})$ & User's y position $(\mathrm{m})$ & User's z position $(\mathrm{m})$ & User's clock time $t_{C}(\mathrm{~s})$ \\
\hline 0 (start) & 0 & 0 & 0 & 37239.9994223656 \\
1 & 5057363.550 & 1986.551 & 3541092.792 & 37239.9975323058 \\
2 & 5226931.564 & -25.990 & 3659938.391 & 37240.0000334559 \\
3 & 5224663.794 & -0.018 & 3658348.973 & 37240.0000000060 \\
4 & 5224663.388 & 0.000 & 3658348.689 & 37240.0000000000 \\
\hline
\end{tabular}

Table 6. Effect of better initialization.

\begin{tabular}{crrrr}
\hline Trial \# & User's x position $(\mathrm{m})$ & User's y position $(\mathrm{m})$ & User's z position (m) & User's clock time $t_{C}(\mathrm{~s})$ \\
\hline 0 (start) & 3313469.280 & -2090568.570 & 5032997.819 & 37240.0043463539 \\
1 & 5190759.859 & 400.888 & 3634586.629 & 37239.9994999186 \\
2 & 5224663.389 & -0.892 & 3658412.593 & 37240.0000000000 \\
3 & 5224663.389 & -0.000 & 3658348.689 & 37240.0000000000 \\
4 & 5224663.389 & 0.000 & 3658348.689 & 37240.0000000000 \\
\hline
\end{tabular}




\section{TIME TAGGING AT THE TRANSMITTER}

Let us now consider time tagging at the transmitters with four satellites. This approach requires a prior estimate of receiver velocity and perhaps acceleration over the interval of signal reception. If all signals leave satellites simultaneously with respect to the ECEF frame, most users will receive these signals within $20 \mathrm{~ms}$ of each other. For most users acceleration will contribute negligibly.

We address two separate issues for this type of receiver. The most important from a conceptual point of view is that, during the $20 \mathrm{~ms}$ or so which may be required for the receiver to make four time-of-arrival measurements, the receiver will generally move through the inertial frame. This motion must be accounted for in order to achieve accurate position determination.

A second, more practical complication, is that the $\mathrm{C} / \mathrm{A}$ code repeats every millisecond, and it might be easiest to choose the GPS system time tag $t_{C}$ for the measurements to be identical to one of the instants at which the $\mathrm{C} / \mathrm{A}$ code begins to repeat. We call this a "code boundary." In the present example we shall illustrate use of $\mathrm{C} / \mathrm{A}$ code in which the receiver only measures the arrival times of code boundaries on its local clock. We shall first discuss C/A code boundary measurements and the necessary time measurement corrections. Then we shall discuss how to account for receiver motion.

\subsection{Code Boundary Measurements}

First we discuss the choice of the time $t_{C}$ at which the ECEF frame is to be "frozen" in place to create the ECIF. The time $t_{C}$ denotes a GPS system time. Unfortunately, because of the clock and relativistic corrections represented by the quantity $\Delta t_{S V}$ in the ICD-GPS-200, the GPS system time $t$ at a satellite differs from the satellite clock time, $t_{S V}$ according to the equation

$$
t=t_{S V}-\Delta t_{S V}
$$

Thus, transmission of a a particular system time $t_{C}$ would occur at satellite clock time which is numerically $\Delta t_{S V}$ later (or earlier if $\Delta t_{S V}$ is negative) by

$$
t_{S V}=t_{C}+\Delta t_{S V}
$$

The correction $\Delta t_{S V j}$ for a particular satellite is contained in the navigation message and can be computed by the receiver. It changes relatively slowly during the $20 \mathrm{~ms}$ or less required by the receiver to make four tinle-of-arrival measurements, so we shall assume that for a particular set of four time-of-arrival measurements, $\Delta t_{S V j}$ are constants.

To assist in making clear the distinctions among the various times which occur in the following discussion, we shall use a subscript $R$ for times measured on the receiver clock, and $S V$ for times measured on the transmitter clock. Both of these may differ from GPS systcun times for events, due to noise, drifts, offsets, and relativistic effects. 


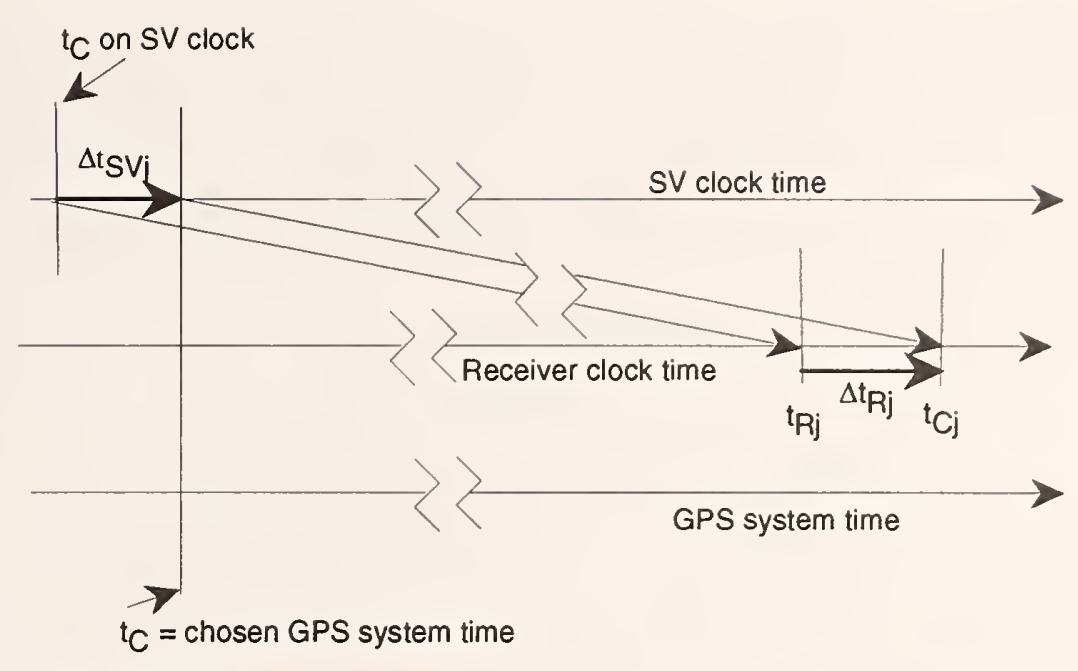

Figure 1. Illustration of SV and receiver clock offsets.

\subsection{Phase Time}

The "phase time" $\tau$ is transmitted from the satellite at SV clock time $t_{S V}$ and propagates to the receiver where it arrives at receiver clock time $t_{R}$. In a particular measurement, $\tau$ is some number. If this number is an integral multiple of $1 \mathrm{~ms}$ so that it lies on a code boundary, then it may be particularly convenient for the receiver to implement the measurement of the arrival time. We shall assume in the following that $\tau$ lies on a code boundary. Thus for the channel corresponding to the $j$ th satellite (with $j=1,2,3,4$ ), the sequence of events may be described as follows.

(a) A common system time $t_{C}$ is chosen for the measurements, which are to be time tagged at the transmitter at this system time $t_{C}$. This system time $t_{C}$ is some integral multiple of $1 \mathrm{~ms}$ and thus there is a code boundary in the phase time which is numerically equal, $\tau=t_{C}$.

(b) While the phase code is numerically equal to $t_{C}$ and is transmitted when the SV clock time $t_{S V j}=\tau=t_{C}$, this does not usually occur at the GPS system time $t=t_{C}$. Because of clock and relativistic corrections, transmission of the true GPS system time $t_{C}$ occurs $\Delta t_{S V j}$ later.

(c) The wave train propagates to the receiver where the code boundary $\tau=$ ${ }^{t_{C}}$ is detected and the receiver clock time $t_{R j}$ of this event is recorded.

This sequence of events is illustrated in Figure 1.

The arrival of a point in the code corresponding to true GPS systenn tine $t_{C}$ will occur an amount $\Delta t_{R j}$ later. Because of the relative nuotion of the receiver and transunitter 
through the ECI, $\Delta t_{R j}$ will in general be different from $\Delta t_{S V j}$ due to the first-order Doppler effect. The receiver needs to estimate this time difference in order to find the arrival time, on the local clock, of the point in the code corresponding to the true time tag $t_{C}$.

The small interval $\Delta t_{R j}$ can be very accurately estimated by using the Doppler formula, eq (D.6) in Appendix D, which relates the coordinate time interval $d t_{R}$ between signal arrivals at the receiver, and the corresponding time interval $d t_{S V}$ at the transmitter

$$
d t_{R}\left(1-\frac{\mathbf{N} \cdot \mathbf{v}}{c}\right)=d t_{S V}\left(1-\frac{\mathbf{N} \cdot \mathbf{V}}{c}\right)
$$

where $\mathbf{V}$ and $\mathbf{v}$ are the transmitter and receiver velocities through the ECI, respectively, at the instants of transmission and reception. This gives the relation between the time interval separating transmission of two nearly equal phase times, and the time interval separating reception of the two phase times. The time intervals $d t_{S V j}$ are less than $1 \mathrm{~ms}$. The first-order Doppler corrections are typically of order $10^{-5}$. Therefore we need good estimates of the velocities of receiver and transmitter, so that the Doppler corrections are known within $10^{-1}$ in order to calculate $d t_{R}$ to better than $1 \mathrm{~ns}$.

Because the Doppler corrections in eq (D.6) are small, we can use eq (D.6) for small time intervals without making appreciable error. Thus, to sufficient accuracy for GPS, eq (D.6) can be rewritten as

$$
\Delta t_{R j}=\Delta t_{S V j} \frac{\left(1-\frac{\mathbf{N}_{j} \cdot \mathbf{v}_{j}}{c}\right)}{\left(1-\frac{\mathbf{N}_{j} \cdot \mathbf{v}}{c}\right)} .
$$

The receiver needs estimates of the receiver velocity $\mathbf{v}$ and the unit vector $\mathbf{N}$ pointing from transmitter to receiver in the ECI. These can be obtained from estimates of position and velocity which have been built up over time during the computation of successive navigation solutions. The transmitter velocity $\mathbf{V}_{j}$ can be computed from the prescriptions given in Table 20-IV in the ICD-GPS-200, by taking derivatives of the satellite position with respect to coordinate time. Since all these vectors must be represented in the chosen ECI, some rotations may be necessary. It is useful to keep in mind, however, that dot products may be computed in any convenient ECI frame.

Thus, in brief, if the phase time corresponding to true GPS system time $t_{C}$ is later than $t_{S V j}=t_{C}$ by $\Delta t_{S V j}$, then the time of reception of the phase time corresponding to $t_{C}$ at the receiver is later by $\Delta t_{R j}$ given by eq (16) above. These time relationships are illustrated in Figure 1.

\subsection{Accounting for Receiver Motion}

In the following discussion we will assume that the code boundary measurements have been made and corrected as described above. After making the necessary corrections, denote the values of the arrival times of the code corresponding to the true GPS time 
$t_{C}$ on the receiver clock by $t_{C j}=t_{R j}+\Delta t_{R j}$. These will still not be GPS system times because in general there will be a bias on the local clock. Denote the bias by $b_{C}$, and denote the GPS system times of arrival by $t_{j}$; then the relation

$$
t_{j}=t_{C j}+b_{C}
$$

holds. Note that there is no subscript $R$ on the time on the left side of eq (17) because this symbol now refers to the GPS system time of an event.

Further, because of the receiver's motion through the ECI, the receiver will not be at a fixed position during the measurements, which occur from 67 to $86 \mathrm{~ms}$ after $t_{C}$ if the receiver is near the earth. This means it is necessary to have available to the receiver an estimate of receiver velocity in the chosen ECI frame, so that the receiver positions at the various reception times can be calculated. Let the receiver position and velocity in the ECI at the system time $t_{C}$ be $\mathbf{r}_{C}$ and $\mathbf{v}_{C}$, respectively. We assume $\mathbf{v}_{C}$ is known to sufficient accuracy, but $\mathbf{r}_{C}$ and $b_{C}$ are to be determined by the measurement. Thus the positions of the receiver at the measurement times are given by

$$
\mathbf{r}_{R j}=\mathbf{r}_{R}\left(t_{j}\right)=\mathbf{r}_{C}+\mathbf{v}_{C}\left(t_{C j}+b_{C}-t_{C}\right) .
$$

The satellite positions at the instant of transmission of the signals at the common GPS system time $t_{C}$ will be denoted by $\mathbf{r}_{j}$. These positions are obtained from the navigation messages and are specified in the WGS-84 reference frame. This means that all of these position vectors are specified in the same reference frame. Therefore it is natural when setting up the propagation delay equations to choose an ECI reference frame which coincides with the ECEF frame at the instant $t_{C}$. Thus no rotations are necessury in order to express these SV positions in a common inertial frame. If we solve for $\mathbf{r}_{C}$, this will be in the ECIF (and the ECEF frame) at $t_{C}$, so no rotation will be required for that vector. However, if we want $\mathbf{r}_{R j}$ in the ECEF frame, rotations.will be necessary.

The propagation delay equations are

$$
\left(\mathbf{r}_{R j}-\mathbf{r}_{j}\right)^{2}=c^{2}\left(t_{j}-t_{C}\right)^{2}
$$

or

$$
\left(\mathbf{r}_{C}+\mathbf{v}_{C}\left(t_{C j}+b_{C}-t_{C}\right)-\mathbf{r}_{j}\right]^{2}=c^{2}\left(t_{C j}+b_{C}-t_{C}\right)^{2} . \quad j=1.2,3.4 .
$$

These four equations can be solved iteratively for the unknown bias $b_{C}$ and the three position components $\mathbf{r}_{C}$ of the receiver in the ECEF frame at the chosen system time $t_{C}$. One iterative method of solution is described in Appendix C. We briefly summarize that process here.

We write the position and bias in tems of estinlates. plus snall corrections:

$$
\mathbf{r}_{C}=\mathbf{r}_{C}^{(i)}+\Delta \mathbf{r} . \quad b_{C}=b_{C}^{(2)}+\Delta t .
$$


Substituting these expressions into eq (19) and expanding, keeping only linear terms in the small quantities $\Delta \mathbf{r}$ and $\Delta t$, leads to a linear system of four equations in four unknowns:

$$
\mathbf{R}_{j}^{(i)} \cdot \Delta \mathbf{r}-c^{2}\left(T_{j}^{(i)}-\mathbf{v}_{c} \cdot \mathbf{R}_{j}^{(i)}\right) \Delta t=\frac{1}{2}\left[c^{2}\left(T_{j}^{(i)}\right)^{2}-\left(\mathbf{R}_{j}^{(i)}\right)^{2}\right],
$$

where

$$
T_{j}^{(i)}=t_{C j}+b_{C}^{(i)}-t_{C},
$$

and

$$
\mathbf{R}_{j}^{(i)}=\mathbf{r}_{C}^{(i)}+\mathbf{v}_{C} T_{j}^{(i)}-\mathbf{r}_{j} .
$$

This system of equations is solved by iteration in the manner described in Appendix C.

\subsection{Time Tagging at the Transmitter-General Prescription:}

We now describe the specific procedure in parallel with the steps described above for time tagging at the receiver.

(1) Measure the times $t_{R j}$ on the local clock at the reception of a given code epoch $\tau$, corresponding to a single GPS phase time $\tau$ in each code train, from each of four satellites.

It may be convenient for a receiver to measure the time on its local clock for the arrival of a particular point of the received code train, such as one of the code boundaries. A code boundary corresponds to a particular millisecond of the week. This millisecond is our chosen time tag. However, each SV clock, and hence the clock's transmitted code, is generally offset from from GPS time, and the offset will be different for each clock. The differences will be less than $1 \mathrm{~ms}$. We will need to account for this in step 2 .

(2) Apply the prescription for corrections as given in the "User Algorithm for SV Clock Correction" of the ICD-200, section 20.3.3.3.3.1, to obtain $\Delta t_{S V j}$

For a given satellite, $\Delta t_{S V}$ is the difference between the instant the code for phase time $\tau$ was transmitted and the value of GPS time $\tau$. (See eqs (14-15).) $\Delta t_{S V}$ includes both the relativistic correction $\Delta t_{r}$ and the broadcast second-order polynomial correction for the SV clock offset from GPS time.

(3) Compute each satellite's position in the ECEF frane at the time of transmission of GPS time $t$.

This is a straightforward application of the equations of Table 20IV, for determining the $x, y, z$ coordinates of the satellite at the GPS time $t_{C}$. 
(4) Choose an ECIF frame for computation of the one-way light times.

In this case the natural choice is to freeze the ECEF frame at the GPS transmission time $t_{C}$.

(5) If the ECI is defined by freezing the ECEF frame at GPS transmission time $t_{C}$, the coordinates of each satellite will not need to be rotated. If we estimate receiver position at $t_{C}$ we do not need to rotate the receiver position coordinates either. We need to adjust the local clock measurements, however, for the time interval $\Delta t_{S V}$ and the motion of the receiver over the time $\Delta t_{S V}$. This is because the code boundary numerically corresponding to $t_{C}$ is not necessarily transmitted at GPS time $t_{C}$.

We may use a deterministic estimate of the change in receiver position over the reception interval. Since this model is usually a velocity, we will refer to it that way here for simplicity. This velocity estimate remains fixed during the solution of user position and time. It is necessary to obtain the velocity estimate in the coordinates of the chosen ECI. Then we nnay estimate receiver coordinates in the ECEF frame which coincides at $t_{C}$ with the ECI. We then use the velocity to estimate receiver positions at the other reception times. We assume the user clock offset from GPS time is constant over the interval of reception.

First apply the corrections represented in eq (16) to compute $\Delta t_{R j}$. Then compute the times of arrival on the local clock $t_{C j}$, of the signal transmitted at GPS time $\tau$. If $t_{R j}$ is the local clock time for the received epoch from satellite $j$, then

$$
t_{C j}=t_{R j}+\Delta t_{R j}
$$

is the local clock time of reception of the signal transmitted at GPS time $t$ from satellite $j$. The correction $\Delta t_{R j}$ is calculated from eq (16) using estimates of $\mathbf{r}_{R j}, \mathbf{r}_{j}$, and $\mathbf{V}_{j}$, and $\mathbf{v}_{j}$ in the ECI which have been built up from a filtering algorithm or from prior solutions.

The time relationships are illustrated in Figure 1. For a given satellite vehicle $S V, \Delta t_{S V}$ is the difference between the instant the code for GPS time $\tau$ was transmitted and the value of GPS time $\tau$. $\Delta t_{S V}$ includes both the relativistic correction $\Delta t_{r}$ and the broadcast second-order polynomial correction for the SV clock offset from GPS time. $\Delta t_{R j}$ is related to $\Delta t_{S V}$ by first-order Doppler corrections given in eq (16).

(6) Solve the equations for the geometric path delays to find the receiver's position and time offset at $t_{C}$.

Again we linearize the propagation delay equations and solve them iteratively. We may iterate to find the position at the first reception 
time, using the velocity estimate to extend to the other reception times. This iteration is similar to step (6) in the case of time tagging at the receiver.

(7) If we have solved for the user position at $t_{C}$ there is no final rotation. In this case the ECIF and ECEF frame coincide at $t_{C}$. If we want the ECEF frame position at a different reception time, a rotation will be required.

It would be possible to update the velocity estimate using positions obtained from two transmission time tags. This leads into other concerns and options associated with using GPS: filtering estimates over time. Techniques for estimating position, velocity, and acceleration could be coupled with strategies for filtering these estimates over time. We will not discuss these options here. We will mention, however, that if there are infrequent measurements of GPS signals from individual satellites, the local clock may be used to flywheel between them and find a solution. This may require careful filtering algorithms for estimation of the local clock frequency. In particular, relativistic effects on the local clock frequency due to velocity or gravitational potential may have to be considered.

\subsection{EXAMPLES}

\section{Example 4.51 Time Tagging at Transmitter}

We give here an example of the analysis of data when the measurements are time tagged at the transmitter. This means that the receiver measures the arrival times of at least four signals which were transmitted simultaneously, at GPS system time $t_{C}$, from four satellites which are labelled with index $j=1,2,3,4$. This method of time tagging has the great advantage that the satellite positions at the instant of transmission, as specified in the navigation message, are all given in the same coordinate system. Thus no rotations are necessary to express these positions in a common inertial frame. For this example we choose a code boundary exactly corresponding to

$$
t_{C}=240.0000000000 \mathrm{~s} .
$$

The following steps are in the order of the seven steps listed in Sections 2 and 3 above.

(1) The times $t_{R j}$ on the local clock of reception of the code epoch $\tau=240$ sec exactly are recorded. These are given in the first column of Table 7 .

(2) The satellite clock corrections $\Delta t_{S V}$, including the relativity corrections, are obtained from the navigation messages; these are given in the second column of Table 7. In this sinulation these offsets are randomly generated within a $1 \mathrm{~ms}$ range using a uniform distribution.

(3) Compute each satellite's position in the ECEF frame at the time of transmission of GPS system time $\tau$. 
This is a straightforward application of the equations of Table 20-IV in the ICD-200, for determining the $x, y, z$ coordinates of the satellites at the GPS time $t_{C}$. The results are given in Table 7 for the four satellites.

(4) Choose an ECI frame for computation of the one-way light times.

In this case the natural choice is to freeze the ECEF frame at the GPS transmission time $t_{C}$.

(5) Compute the received time offsets $\Delta t_{R j}$ and the times $t_{C j}$ of arrival on the local clock, of the signals transmitted at GPS system time $t_{C}=\tau$.

We must make the first-order Doppler corrections, then compute the time intervals $\Delta t_{R j}$. This requires estimating the Doppler correction. However since the Doppler corrections are very small, in a first approximation we might neglect the Doppler correction factor entirely and assume $\Delta t_{R j} \approx \Delta t_{S V j}$. We might then use the resultant positions to estimate $\mathrm{N}_{j}$ to within a few percent. If the estimate of position in the chosen ECIF is within $890 \mathrm{~km}$ of the true position and the receiver is near the earth, then the error made in estimating the direction cosines of the line-of-sight unit vector $\mathrm{N}_{j}$ will then be less than $10^{-3}$. In a second approximation, estimates of the Doppler correction factor which use velocities and a value of $\mathbf{N}_{j}$ accurate within a few percent should be adequate.

Estimating receiver velocity. In the present example we consider a receiver which has an estimated ground velocity of $v_{g}=300 \mathrm{~m} / \mathrm{s}$ in a direction $30^{\circ}$ north of east, parallel to earth's surface. Suppose the receiver at the instant $t_{C}=240 \mathrm{~s}$ exactly is at the position

$$
\mathbf{X}_{\text {WGS84 }}=\left[\begin{array}{c}
R \cos 35^{\circ} \\
0 \\
R \sin 35^{\circ}
\end{array}\right]=\left[\begin{array}{c}
5224663.389 \mathrm{~m} \\
0 \\
3658348.690 \mathrm{~m}
\end{array}\right] \text {. }
$$

which has been used in our earlier examples. The net velocity will be a linear combination of the ground velocity and velocity of the point $\mathrm{X}_{W G S 84}$ due to earth rotation. From geometric considerations, the net receiver velocity at time $t_{C}$ in this example will be

$$
\mathbf{v}_{C}=\left[\begin{array}{c}
-v_{g} \sin 30^{\circ} \sin 35^{\circ} \\
v_{g} \cos 30^{\circ}+\dot{\Omega}_{e} R \cos 35^{\circ} \\
v_{g} \sin 30^{\circ} \cos 35^{\circ}
\end{array}\right] .
$$

If the position and ground velocity were accurately known. the net receiver velocity in the ECIF would then be

$$
\mathbf{v}_{C}=\left[\begin{array}{rrr}
-86.036 & 465 & 453 \\
640.796 & 091 & 481 \\
122.872 & 806 & 643
\end{array}\right] \mathrm{m} / \mathrm{s} .
$$


Therefore in the present example, we take the velocity estimate in the ECIF to be

$$
\mathbf{v}_{C}=\left[\begin{array}{r}
-86 \\
641 \\
123
\end{array}\right] \mathrm{m} / \mathrm{s} .
$$

Estimating receiver position. We illustrate the calculation of the first-order Doppler correction with satellite 1 in Table 7. The unit vector $\mathrm{N}$ is computed from the satellite and receiver positions, as in eq (D.3):

$$
\mathbf{N}=\frac{\mathbf{r}_{R}\left(t_{R}\right)-\mathbf{r}_{T}\left(t_{T}\right)}{\left|\mathbf{r}_{R}\left(t_{R}\right)-\mathbf{r}_{T}\left(t_{T}\right)\right|}
$$

where $\mathbf{r}_{R}\left(t_{R}\right)$ and $\mathbf{r}_{T}\left(t_{T}\right)$ are the ECIF position vectors of receiver and transmitter at reception and transmission times $t_{R}$ and $t_{T}$. At the receive time, with bias of correction $423 \mathrm{~ns}$, the receiver position vector is actually

$$
\begin{aligned}
\mathbf{r}_{R 1}=\mathbf{r}_{C}+\mathbf{v}_{C}\left(240.0691241403-0.0000004230-t_{C}\right) \\
=\left[\begin{array}{c}
5224656.660 \\
50.117 \\
3658358.300
\end{array}\right] \mathrm{m},
\end{aligned}
$$

and we therefore take as an estimate of this position the vector

$$
\mathbf{r}_{R 1} \approx\left[\begin{array}{ccc}
6 & 000 & 000 \\
23 & 000 \\
3 & 100 & 000
\end{array}\right] \mathrm{m}
$$

and the receiver velocity is given in eq (28), which was discussed above. The bias correction was not significant in arriving at this rough estimate of receiver position.

Estimating satellite velocity. The satellite position at transmit time $t_{C}$ is given in the first row of Table 7. The satellite velocity is also needed. It may be obtained by differentiating the satellite positions, which are derivable from the prescription of Table 20-IV in the ICD-GPS-200, with respect to time, and then applying a rotation into the ECIF. For the present example the satellite velocity in the ECIF is

$$
\mathrm{V}_{1}=\left[\begin{array}{ccc}
-304.267 & 760 \\
3 & 853.051 & 168 \\
-257.220 & 637
\end{array}\right] \mathrm{m} / \mathrm{s} \text {. }
$$


The position estimates result in the following calculated values of the components of $\mathrm{N}_{1}$ :

$$
\mathrm{N}_{1} \approx\left[\begin{array}{ll}
-0.437 & 588 \\
-0.114 & 125 \\
-0.891 & 904
\end{array}\right]
$$

while the "true" value of $\mathrm{N}_{1}$ obtained from the simulation is

$$
\mathrm{N}_{1}=\left[\begin{array}{lll}
-0.477 & 846 & 083 \\
-0.115 & 974 & 357 \\
-0.870 & 754 & 311
\end{array}\right]
$$

For the present case, $\Delta t_{S V}=-0.0000565547834 \mathrm{~s}$. Then eq (16) gives the estimate

$$
\Delta t_{R 1} \approx-0.000056554784 \mathrm{~s} .
$$

This estimate agrees extremely well with the "true" value of $\Delta t_{R 1}$ obtained from the simulation. For details of this comparison, see Appendix E.

Thus the Doppler correction factor given in eq (16) can be computed by the receiver and added to $t_{R j}$ to predict, with excellent accuracy, the arrival time $t_{R j}+d t_{R j}$ of the code which corresponds to transmission of $t_{C}$ from the satellite. Thus only times of arrival of code boundaries would actually be measured by the receiver.

Three additional calculations using eq (16) are given in the third column of Table 7 for the satellites used in the present example. Here the time $t_{C}$ equals $240.000000000 \mathrm{~s}$ exactly. The first column of Table 7 gives local clock arrival times of the phase time $\tau=t_{C}$. The second column lists the values of the SV clock and relativistic corrections derived from the navigation messages. The third column lists the corrections $\Delta t_{R j}$ obtained from eq (16). Note that the largest difference between $\Delta t_{S V j}$ and $\Delta t_{R j}$ is little more than $0.5 \mathrm{~ns}$. The maximum possible such difference is about $4 \mathrm{~ns}$ and could occur for a clock in a satellite in low earth orbit. The resulting values of local clock arrival times $t_{C j}$ are listed in the last colunn of Table 7 and are repeated in the last column of Table 8. There may still be a local clock bias in these data. In this example, the receiver clock has a bias of $-423 \mathrm{~ns}$. 
Table 7. Computation of first-order Doppler corrections.

$$
\text { (units }=\mathrm{s} \text { ) }
$$

\begin{tabular}{ccccc}
\hline ID \# & \multicolumn{1}{c}{$t_{R j}$} & $\Delta t_{S V j}$ & $\Delta t_{R j}$ eq $(16)$ & $t_{C j}=t_{R j}+\Delta t_{R j}$ \\
\hline 1 & 240.069180695048 & -0.0000565547962 & -0.000056554784 & 240.069124140263 \\
2 & 240.078615674081 & 0.0004937927006 & 0.000493792194 & 240.079109466275 \\
3 & 240.075360518523 & 0.0002597259299 & 0.000259725401 & 240.075620243923 \\
4 & 240.072939857576 & 0.0003057143767 & 0.000305713988 & 240.073245571564 \\
\hline
\end{tabular}

Table 8. Satellite positions $\mathbf{r}_{j}$ and measured arrival times $t_{C j}$.

\begin{tabular}{crrrr}
\hline ID \# & Satellite $x_{j}(\mathrm{~m})$ & Satellite $y_{j}(\mathrm{~m})$ & Satellite $z_{j}(\mathrm{~m})$ & Local clock time $t_{C j}(\mathrm{~s})$ \\
\hline 1 & 15126951.488 & 2403354.115 & 21702797.709 & 240.0691241403 \\
2 & 20996573.361 & -15349939.783 & -5178329.880 & 240.0791094663 \\
3 & 23391384.147 & 10956612.394 & -4333140.777 & 240.0756202439 \\
4 & 10656983.742 & -11270652.822 & 21703578.299 & 240.0732455716 \\
\hline
\end{tabular}

Table 8 gives the data from the all four measurements: the derived positions of the satellites at GPS system transmission times and the corresponding arrival times $t_{C j}$ on the receiver clock.

(6) The iterative solution, which has been carried out as described in Appendix $\mathrm{C}$ is given at successive stages in Table 9. At the initial stage, estimates for the ECEF receiver position are inserted. For the sake of illustration, we assume the initial estimate of the receiver position places it at earth's center. The value of the local clock bias is initially estimated to be $300 \mathrm{~ns}$. The iteration process converges rapidly, to within a millimeter or so of the correct ECEF receiver position.

(7) No rotations are necessary in the final stage since the position has been determined in the ECEF frame.

The receiver positions at the times $t_{C j}$ of reception might be needed for other purposes. These could be obtained using a best estimate of the velocity of the receiver in the ECIF (the ECEF frame at $t_{C}$ ) using eq (18). The components of $\mathbf{r}_{C}$ are in this reference frame. A second series of measurements at a slightly later time $t_{C}^{\prime}$ will give a new value of $\mathbf{r}_{C}$ in the new reference frame. These two values could be differenced or filtered to provide an update to the ground velocity. Such an updated value could be transformed into an ECIF 
Table 9. Iteration solution for $\mathbf{r}_{C}$ and receiver clock bias $b_{C}$.

\begin{tabular}{ccccc}
\hline Trial \# & Receiver $x_{C}(\mathrm{~m})$ & Receiver $y_{C}(\mathrm{~m})$ & Receiver $z_{C}(\mathrm{~m})$ & Local clock bias $b_{C}(\mathrm{~s})$ \\
\hline 0 & 0.0 & 0.0 & 0.0 & 0.0000003000 \\
1 & 5051460.568 & -9835.265 & 3545131.610 & -0.0024534252 \\
2 & 5226991.162 & 132.182 & 3659870.280 & 0.0000325443 \\
3 & 5224663.799 & -0.023 & 3658349.573 & -0.0000004172 \\
4 & 5224663.390 & -0.000 & 3658348.690 & -0.0000004230 \\
5 & 5224663.390 & -0.000 & 3658348.690 & -0.0000004230 \\
\hline
\end{tabular}

with the help of a rotation such as in eq (28), to be used in further position measurements.

\section{Example 4.52 Time Tagging at Transmitter Neglecting Doppler Corrections}

The first-order Doppler corrections to $\Delta t_{S V}$ are very small, as seen in Example 4.51, above. Here we repeat Example 4.51 with one change: the Doppler corrections are completely neglected. Since including these first-order Doppler corrections usually amount to changing the arrival times on the local clock by less than 1 ns, only a few centimeters of error would be expected to occur when such corrections are neglected. Table 10 gives the satellite positions $\mathbf{r}_{j}$ and the measured arrival times $t_{C j}$ when this approximation is made. The table is identical to Table 8 except in the fourth column.

Solutions of Eqs. (21) by iteration are given in Table 11. Thus neglecting the firstorder Doppler correction causes a position error of about $75 \mathrm{~cm}$ in this case.

Table 10. Satellite positions $\mathbf{r}_{j}$ and arrival times $t_{C j}$ neglecting Doppler correction.

\begin{tabular}{crrrr}
\hline ID \# & satellite $x_{j}(\mathrm{~m})$ & satellite $y_{j}(\mathrm{~m})$ & satellite $z_{j}(\mathrm{~m})$ & $t_{C j} \approx t_{R j}+\Delta t_{S V j}(\mathrm{~s})$ \\
\hline 1 & 15126951.488 & 2403354.115 & 21702797.709 & 240.0691241403 \\
2 & 20996573.361 & -15349939.783 & -5178329.880 & 240.0791094668 \\
3 & 23391384.147 & 10956612.394 & -4333140.777 & 240.0756202445 \\
4 & 10656983.742 & -11270652.822 & 21703578.299 & 240.0732455720 \\
\hline
\end{tabular}

\section{TIME TRANSFER RECEIVER}

In this case we may use each pseudorange measurement from each satellite separately to estimate our clock offset from GPS time. We either time tag measurements at a transmission time and measure time of reception, or time tag measurenents at a reception time and determine time of transmission from the code lock of the receiver. We then use the 
Table 11. Solution for $\mathbf{r}_{C}$ and receiver clock bias $b_{C}$ neglecting Doppler correction.

\begin{tabular}{ccccc}
\hline Trial \# & Receiver $x_{C}(\mathrm{~m})$ & Receiver $y_{C}(\mathrm{~m})$ & Receiver $z_{C}(\mathrm{~m})$ & Local clock bias $b_{C}(\mathrm{~s})$ \\
\hline 0 & 0.0 & 0.0 & 0.0 & 0.0000003000 \\
1 & 5051461.207 & -9835.359 & 3545131.862 & -0.0024534276 \\
3 & 5226991.866 & 132.086 & 3659870.566 & 0.0000325424 \\
4 & 5224664.501 & -0.072 & 3658349.243 & -0.0000004191 \\
5 & 5224664.092 & -0.096 & 3658348.976 & -0.0000004249 \\
6 & 5224664.092 & -0.096 & 3658348.976 & -0.000000424 .9 \\
\hline
\end{tabular}

prescriptions from the ICD-200, Table 20-IV, to obtain the satellite position in the ECEF frame. We must estimate the geometric path delay $t_{D}$ in addition to the ionospheric and tropospheric corrections. Since we know the receiver position we may compute $t_{D}$ as follows.

If ECI position vectors are referenced to the time of signal transmission, then

$$
t_{D}=t_{R}-t_{T}=\frac{\left|\mathbf{r}\left(t_{R}\right)-\mathbf{R}\left(t_{T}\right)\right|}{c} \approx \frac{\left|\mathbf{r}\left(t_{T}\right)-\mathbf{R}\left(t_{T}\right)\right|}{c}+\frac{\left[\mathbf{r}\left(t_{T}\right)-\mathbf{R}\left(t_{T}\right)\right] \cdot \mathbf{v}}{c^{2}},
$$

where

$\mathbb{R}$ is the $\mathrm{SV}$ position vector,

$\mathbf{r}$ is the receiver position vector,

$t_{T}$ and $t_{R}$ are GPS time at respectively transmit and receive times, and $\mathrm{v}$ is the receiver velocity vector in the ECIF.

The last term in eq (34) is sometimes called the Sagnac correction. In the case of an earth-fixed user,

$$
\mathbf{v}=\dot{\Omega}_{e} \times \mathbf{r} .
$$

If ECIF position vectors are referenced to the time of signal reception (instead of satellite transmission time), then

$$
t_{D}=t_{R}-t_{T}=\frac{\left|\mathbf{r}\left(t_{R}\right)-\mathbf{R}\left(t_{T}\right)\right|}{c} \approx \frac{\left|\mathbf{r}\left(t_{R}\right)-\mathbf{R}\left(t_{R}\right)\right|}{c}-\frac{\left[\mathbf{r}\left(t_{R}\right)-\mathbf{R}\left(t_{R}\right)\right] \cdot \mathbf{V}}{c^{2}}
$$

where:

$$
\mathrm{V} \text { is the SV velocity vector in the ECI. }
$$

A single-channel receiver with antenna placed at a known location can provide a means for accurate transfer of GPS time to a local clock. Let the antenna be at the position $\mathbf{r}_{E C E F}$ whose earth-fixed coordinates are accurately known and do not change with time. 
The appropriate expression for the total propagation delay for a signal from one satellite is eq (34). In eq (34) the positions are ECI positions but are referred to the time of transmission $t_{T}$. The first term on the right side of eq (34) is proportional to the distance between receiver and transmitter at the transmit time $t_{T}$; if we call this distance $L$, then

$$
L=\left|\mathbf{r}\left(t_{T}\right)-\mathbf{R}\left(t_{T}\right)\right|
$$

Although the position vectors $\mathbf{r}\left(t_{T}\right)$ and $\mathbf{R}\left(t_{T}\right)$ are ECI position vectors, the distance $L$ is invariant under spatial rotation and can therefore be computed in any convenient coordinate system. The ECEF position of the satellite at transmit time is directly derivable from the prescription given in the ICD-200, Table 20-IV, and therefore no rotations are necessary to compute $L$ :

$$
L=\left|\mathbf{r}_{E C E F}\left(t_{T}\right)-\mathbf{R}_{E C E F}\left(t_{T}\right)\right| .
$$

The second term in eq (34), the Sagnac correction term, can be further reduced for this case since it also is a scalar with respect to rotations. The ECI velocity of the earth-fixed receiver is due to earth rotation and is

$$
\mathbf{v}=\dot{\Omega}_{e} \times \mathbf{r}\left(t_{T}\right) .
$$

The Sagnac correction term, which we denote by $\Delta t_{\text {Sagnac }}$, is therefore

$$
\begin{aligned}
\Delta t_{\text {Sagnac }} & =\frac{\left[\mathbf{r}\left(t_{T}\right)-\mathbf{R}\left(t_{T}\right)\right] \cdot \dot{\Omega}_{e} \times \mathbf{r}\left(t_{T}\right)}{c^{2}} \\
& =\frac{\dot{\Omega}_{e} \cdot \mathbf{r}\left(t_{T}\right) \times\left[\mathbf{r}\left(t_{T}\right)-\mathbf{R}\left(t_{T}\right)\right]}{c^{2}} \\
& =\frac{\dot{\Omega}_{e} \cdot \mathbf{r}\left(t_{T}\right) \times\left[-\mathbf{R}\left(t_{T}\right)\right]}{c^{2}} \\
& =\frac{\dot{\Omega}_{e} \cdot \mathbf{R}\left(t_{T}\right) \times \mathbf{r}\left(t_{T}\right)}{c^{2}} \\
& =\frac{\dot{\Omega}_{e} \cdot \mathbf{R}_{E C E F}\left(t_{T}\right) \times \mathbf{r}_{E C E F}\left(t_{T}\right)}{c^{2}} \\
& =\frac{\left|\dot{\Omega}_{e}\right|\left(X_{E C E F}\left(t_{T}\right) y_{E C E F}\left(t_{T}\right)-Y_{E C E F}\left(t_{T}\right) x_{E C E F}\left(t_{T}\right)\right)}{c^{2}},
\end{aligned}
$$

where standard vector operations have been used to simplify the scalar product. In the last step, the only component of the cross product which contributes is parallel to the earth's angular velocity vector; this product is invariant under rotations about this axis. so the computation can be performed in the ECEF frame at the instant of transmission. Thus for a timing receiver no rotations need be applied to compute the local time. 
The final result in eq (40) above can be put in a form which is easy to interpret and can be found in many places in the literature:

$$
\Delta t_{\text {Sagnac }}=\frac{2\left|\dot{\Omega}_{e}\right|}{c^{2}} A_{E}
$$

where $A_{E}=\frac{1}{2}\left[\mathrm{R}\left(t_{T}\right) \times \mathbf{r}\left(t_{T}\right)\right]_{z}$ is the equatorial projection of the area swept out by a vector from the center of the earth to the path of the timing pulse as it propagates from transmitter at $\mathbf{R}\left(t_{T}\right)$ to receiver at $\mathbf{r}\left(t_{T}\right)$. Clearly this area can be calculated in either ECIF or ECEF coordinates.

\section{Example 5.1 Earth-fixed Single-Channel Timing Receiver}

We give four examples here based on the data from four different satellites given in Table 1. For all these examples the ECEF position of the receiver is that specified in eq (7).

In Table 12 the first column labels different satellites corresponding to the data entries in Table 1. The second column gives the transmission epochs. The third column gives the transmission delay contributions $L / c$, the fourth column gives the Sagnac corrections, and the fifth column sums the entries in the previous three columns to give the time at the receiver. The agreement of the entries in the last column reflects consistency of the procedure across the satellite constellation.

Table 12. Examples of time transfer to a single-channel earth-fixed receiver.

\begin{tabular}{ccccc}
\hline SV \# & Transmission epoch $t_{T}(\mathrm{~s})$ & $L / c(\mathrm{~s})$ & $\Delta t_{\text {Sagnac }}(\mathrm{s})$ & $t_{R j}(\mathrm{~s})$ \\
\hline 1 & 37239.9244223656 & 0.0755777148 & -0.0000000805 & 37240.0000000000 \\
2 & 37239.9207133918 & 0.0792866775 & -0.0000000855 & 37240.0000000000 \\
3 & 37239.9253078700 & 0.0746921974 & -0.0000000674 & 37240.0000000000 \\
4 & 37239.9293463539 & 0.0706536092 & 0.0000000369 & 37240.0000000000 \\
\hline
\end{tabular}

\section{REFERENCES}

[1] "Navstar GPS Space Segment/Navigation User Interfaces," ICD-GPS-200, Revision C (10 October 1993), Code Ident. No. 29562, ARINC Research Corporation, 4055 Hancock Street, San Diego, CA 92110.

[2] Ashby, N.; J.J. Spilker, Jr., "Introduction to Relativistic Effects in the Global Positioning System," Ch. 18 in Global Positioning System: Theory and Applications, Vol. I, eds., B. W. Parkinson and J. J. Spilker, Jr., Progress in Astronautics and Aeronautics, 
Vol. 163, AIAA, Inc., 370 L'Enfant Promenade, SW, Washington, DC 20024-2518 (1996).

[3] Ashby, N. "Relativistic Effects in the Global Positioning System," Chinese J. Systems Eng. Elect. 6, 199-237 (1995).

[4] Gibson, L. Ralph, "A Derivation of Relativistic Effects in Satellite Tracking," Naval Surface Weapons Center TR 83-55, (April 1983).

[5] Deines, S., "Missing Relativity Effects in GPS," paper presented at ION-GPS-90, Colorado Springs, CO, September 1990. See also "Missing Relativity Terms in the Current GPS Algorithm," S. Deines, Contract F04701-87-C-0027, (June 1990), and "Missing Relativity Terms in the GPS," Navigation 39, 111-131 (1992).

[6] Fliegel, H.; R. DiEsposti (1997), "GPS and Relativity: an Engineering Overview," Aerospace Report ATR-97(3389-1).

[7] "Department of Defense World Geodetic System 1984, Its Definition and Relationships with Local Geodetic Systems," DMA Technical Report 8350.2, Stock Number DMATR83502WGS84, U.S. Geological Survey, Denver, CO, 80225.

[8] Malys, S.; J. Slater, "Maintenance and Enhancement of the World Geodetic System 1984" (1994), Proc. ION-GPS-94, Salt Lake City, Sept. 20-23, 17-24 (1994).

[9] "1992 IERS Annual Report" (July 1993), Central Bureau of IERS, Observatoire de Paris, 61 avenue de l'Observatoire, F-75014 Paris, France.

[10] "IERS Standards (1989)," D.D. McCarthy, ed., IERS Technical Note No. 3, Observatoire de Paris, 61 avenue de l'Observatoire, F-75014 Paris, France.

[11] Moritz, H.; I.I. Meuller (1987), Earth Rotation, Theory and Observation, Ungar, New York.

[12] Hofmann-Wellenhof, B.; H. Lichtenegger, J. Collins (1993), GPS Theory and Practice, Second Edition, Springer-Verlag, New York.

[13] Goldstein, H., Classical Mechanics, 2nd Ed. (1980), Addison-Wesley Publishing, Reading, MA, Sect. 4-9, p. 174.

[14] "Relativistic Effects in the Global Positioning System," D. Eardley et al., JASON. MITRE Corporation, 1820 Dolley Madison Blvd, McLean, VA 22102, May 1985.

[15] "Accuracy of Time Transfer in Satellite Systems," Committee on Accuracy of Time Transfer in Satellite Systems, Air Force Studies Board, National Academy Press. Washington, D.C. (1986).

[16] See eq (2) of "Lectures on Frequency Stability and Clocks," by R.F.C. Vessot. in 
Experimental Gravitation, ed. B. Bertotti, Academic Press, Inc., 155 (1974).

[17] Boucher, C.; Z. Altamimi, M. Feissel, P. Sillard, "Results and Analysis of the ITRF94," IERS Technical Note 20, Central Bureau of IERS (March 1996), Observatoire de Paris, 61, avenue de l'Observatoire, F-75014 PARIS, France. 


\section{Appendix A.}

\section{The Eccentricity Correction}

The epoch of the transmission event from each satellite must be corrected to account for variations in the proper time of each satellite clock due to orbital eccentricity. This aspect of system design of the GPS was fixed very early when satellites did not have much computing power. The correction compensates the atomic clock in the satellite for periodic gravitational frequency shifts and second-order Doppler shifts due to the satellites' periodic change of radius and velocity in its eccentric orbit. The correction converts the proper time kept on the atomic clock, to coordinate time-that is, to GPS time. To put it another way, a satellite in an eccentric orbit samples different values of earth's gravitational field as the satellite moves toward and away from the earth. Consequently it suffers a periodic gravitational frequency shift. At the same time, conservation of angular momentum causes the satellite to speed up or slow down, giving rise to a periodic second-order Doppler shift. Thus SV clocks must be corrected for these effects so that the corrected clocks effectively run at a constant rate, the rate of coordinate time.

It is important to realize that this correction is the same for all receivers, no matter where they are or what their state of motion is. The correction relates solely to the transmitter. In particular it is correct for users of GPS anywhere in space. It is incorrect to claim, as is done in Ref. [6], that the correction applies only to users near earth's surface.

Let us denote the time that the user estimates from the information encoded in the signal as $t_{S V}$ as in the ICD-GPS-200. This must be corrected according to the section describing the user algorithm to obtain GPS system time $t$ (Sect. 20.3.3.3.3.1 in the ICDGPS-200C). The correction has several different, but equivalent forms. Using the notation of ICD-GPS-200C, Sect. 20.3.3.3.3.1, the relativistic correction to the transmission epoch $t_{S V}$ is

$$
\begin{aligned}
\Delta t_{r} & =-2 \frac{\sqrt{G M A}}{c^{2}} e \sin E_{k} \\
& =F e \sqrt{A} \sin E_{k} \\
& =-\frac{2 \mathbf{R} \cdot \mathbf{V}}{c^{2}}
\end{aligned}
$$

where

$$
F=-4.442807633 \times 10^{-10} \mathrm{~s} / \sqrt{\mathrm{m}},
$$

and where $G M$ is the product of the Newtonian gravitational constant and the mass of the earth, $A$ is the semi-major axis of the satellite in meters, $e$ is the eccentricity, $E_{k}$ is the satellite's eccentric anomaly, $\mathbf{R}$ is the instantancous position vector of the SV at transmission time, and V is the instantaneous velocity vector of the SV. We need GPS 
time $t$ before we can obtain $E_{k}$ as prescribed in Table 20-IV, so the equations are coupled. It suffices to use $t_{S V}$ to determine $E_{k}$, then obtain GPS time $t$. Also, since the scalar product in the last form of the correction is invariant under spatial rotations, it can be evaluated in any inertial frame. This correction $\Delta t_{r}$ is subtracted from the transmitted time $t_{S V}$ to obtain GPS system time $t$. The time $t$ may then be applied to the user algorithm for ephemeris (Table 20-IV in the ICD-GPS-200C), to obtain the $x, y, z$ coordinates of each satellite at transmission time.

Derivations of the above results can be found in references [2], [3], and [4]. 


\section{Appendix B.}

\section{Linearization Algorithm for Time Tagging at Receiver}

We now explain one possible method of linearization of the one-way light time equations which are valid in an inertial coordinate system.

Consider four GPS satellites which at GPS times $t_{1}, t_{2}, t_{3}, t_{4}$ send out signals from the ECIF locations $\mathbf{r}_{1}, \mathbf{r}_{2}, \mathbf{r}_{3}, \mathbf{r}_{4}$. We assume that these data have been expressed in the chosen ECI reference frame. These four signals are received simultaneously at time $t$ by a receiver at position $\mathbf{r}$. The problem is to determine $t$ and $\mathbf{r}$ at the receiver. The velocity of the receiver does not have to be considered. The receiver's position $\mathbf{r}$ at the time of reception $t$ will be determined by the solution of four equations which express the condition that the speed of propagation is $c$. The four equations are

$$
\left(\mathbf{r}-\mathbf{r}_{j}\right)^{2}-c^{2}\left(t-t_{j}\right)^{2}=0 ; \quad j=1,2,3,4
$$

Such equations are clumsy to solve in general. However, if the position $\mathbf{r}$ and time $t$ are known approximately, the equations can be reduced to a system of four linear equations which can be solved by standard matrix inversion techniques.

Suppose that for user position and time we write

$$
\mathbf{r}=\mathbf{r}^{(i)}+\Delta \mathbf{r} ; \quad t=t^{(i)}+\Delta t
$$

where the quantities $\mathbf{r}^{(i)}, t^{(i)}$ represent guesses or trial values for the position and time, and $\Delta \mathbf{r}, \Delta t$ are assumed to be small quantities which are to be determined by solving eqs (B.1). The index $i$ in eq (B.2) labels the $i$ th trial value in a repeated or iterative process of solving Eqs. (B.1).

We substitute eqs (B.2) into eqs (B.1), expand and neglect squares of the small quantities $\Delta \mathbf{r}$ and $\Delta t$. Then the equations take the following form

$$
\left(\mathbf{r}^{(i)}-\mathbf{r}_{j}\right) \cdot \Delta \mathbf{r}-c^{2}\left(t^{(i)}-t_{j}\right) \Delta t=\frac{1}{2}\left(c^{2}\left(t^{(i)}-t_{j}\right)^{2}-\left(\mathbf{r}^{(i)}-\mathbf{r}_{j}\right)^{2}\right),
$$

for $j=1,2,3,4$. In eqs (B.3), the quantity $c\left(t^{(i)}-t_{j}\right)$ is the $i$ th estimate of the pscudorange from the receiver to the $j$ th satellite. Eqs (B.3) are a system of linear inhomogeneous equations which can be solved by matrix inversion. Numerical examples of the solution are discussed in the main text. The matrix of coefficients of the unknowns $\Delta \mathrm{r}$ and $\Delta t$ will usually be nonsingular, unless the configuration of satellites is so unfavorable that eqs (B.1) do not have a solution. The solutions obtained will be approximate. but can be used to obtain new trial values; the process of iteration can then be repeated. 
Thus, suppose at stage $i$ in the iteration, eqs (B.3) have been solved for corrections $\Delta \mathrm{r}$ and $\Delta t$; then better approximations can be found by setting

$$
\mathbf{r}^{(i+1)}=\mathbf{r}^{(i)}+\Delta \mathbf{r} ; \quad t^{(i+1)}=t^{(i)}+\Delta t .
$$

The iteration converges rapidly, particularly if the initial trial guesses were close. The solutions will be found at some stage when the inhomogeneous terms on the right sides of eqs (B.3) become negligible. Then the solutions satisfy

$$
\left(\mathbf{r}^{(i)}-\mathbf{r}_{j}\right)^{2}-c^{2}\left(t^{(i)}-t_{j}\right)^{2}=0 ; \quad j=1,2,3,4,
$$

which are equivalent to eqs (B.1).

In summary the computation scheme proceeds as follows:

Step 1. Choose approximate values for user position and time.

Step 2. Solve eqs (B.3) to obtain corrections $\Delta \mathbf{r}$ and $\Delta t$ for user position and time.

Step 3. Test whether the corrections are sufficiently small that computation can be halted; if not, combine the corrections obtained in Step 2 with the approximate values of position to obtain a new approximate position. Then go back to Step 2. 


\section{Appendix C.}

\section{Linearization Algorithm for Time Tagging at Transmitters}

When the measurements are time tagged at the transmitters, then the measurements at the receiver will occur at unequal times. This entails a different method for linearization of the propagation delay equations than that discussed in Appendix B.

Suppose that we decide to time tag the signals from four GPS satellite transmitters at the common GPS system time $t_{C}$. Let the arrival times of these signals on the receiver clock be $t_{R j}$, and let the positions of the satellites at time $t_{C}$ from which these signals were transmitted be $\mathbf{r}_{j}$, with $j=1,2,3,4$. These positions are derived from the navigation messages; they will be expressed in the ECEF frame at the instant $t_{C}$. Therefore it is highly advantageous to work with an ECI reference frame which coincides with the ECEF frame as though it were frozen at $t_{C}$. Then no rotations will be necessary to bring the satellite positions into a common ECI frame-they are already expressed in the ECIF defined at $t_{C}$. If we solve the propagation delay equations for the receiver position at $t_{C}$ we have the receiver position in this same ECIF. Thus time tagging at the transmitters . has the great advantage that no rotations are needed.

In general the signal propagation times from satellites to receiver will not be equalthey can vary roughly from 67 to $86 \mathrm{~ms}$ for a receiver on earth's surface. This means that it will be necessary to have an estimate of the receiver velocity at the time $t_{C}$ in order that the different receiver positions at the different signal arrival times can be accounted for. We may also need the receiver's acceleration. We assume these are available, to sufficient accuracy, from previous position solutions or from a filtering algorithm.

We will illustrate this here by assuming the receiver position is expressible as a Taylor series

$$
\mathbf{r}_{R}(t)=\mathbf{r}_{C}+\mathbf{v}_{C}\left(t-t_{C}\right)+\ldots,
$$

where $\mathbf{r}_{C}$ and $\mathbf{v}_{C}$ are the receiver position and velocity in the chosen ECIF, at time $t_{C}$. An additional acceleration term in this expansion might be needed for a receiver in a highly dynamic trajectory. We assume here that $\mathbf{v}_{C}$ is known but $\mathbf{r}_{C}$ is unknown.

The measured arrival times $t_{R j}$ of the signals on the receiver clock will in general not be true GPS system times because of a possible bias $b_{C}$ on the receiver clock. Thus the GPS system times of arrival will be

$$
t_{j}=t_{R j}+b_{C},
$$

and as part of the data analysis, the bias will be found. The receiver positions at times $t_{j}$ will therefore be

$$
\mathbf{r}_{R}\left(t_{j}\right) \equiv \mathbf{r}_{R j}=\mathbf{r}_{C}+\mathbf{v}_{C}\left(t_{R j}+b_{C}-t_{C}\right)
$$


In this Appendix we do not discuss code boundary measurements and corrections. This important topic is discussed in detail in Sect. 3. We simply assume that the appropriate places in the code have been identified by the receiver.

The four propagation delay equations are then

$$
\left(\mathbf{r}_{R j}-\mathbf{r}_{j}\right)^{2}=c^{2}\left(t_{R j}+b_{C}-t_{C}\right)^{2}
$$

or

$$
\left(\mathbf{r}_{C}+\mathbf{v}_{C}\left(t_{R j}+b_{C}-t_{C}\right)-\mathbf{r}_{j}\right]^{2}=c^{2}\left(t_{R j}+b_{C}-t_{C}\right)^{2} .
$$

The unknown position $\mathbf{r}_{C}$ and bias $b_{C}$ may be found from these four equations as follows. As in Appendix B, we assume we can write the unknown quantities in terms of good estimates, plus small corrections. Thus we write

$$
\mathbf{r}_{C}=\mathbf{r}_{C}^{(i)}+\Delta \mathbf{r}, \quad b_{C}=b_{C}^{(i)}+\Delta t
$$

where the quantities $\mathbf{r}_{C}^{(i)}, b_{C}^{(i)}$ represent trial estimates for the position vector and clock bias, and $\Delta \mathbf{r}, \Delta t$ are small quantities. The index $(i)$ in eq (C.6) labels the $i^{t h}$ trial value in a repeated or iterative process of solving eqs (C.5).

We substitute (C.6) into (C.5), expand and neglect squares of small quantities $\Delta \mathbf{r}$ and $\Delta t$. Then the equations reduce to

$$
\mathbf{R}_{j}^{(i)} \cdot \Delta \mathbf{r}-c^{2}\left(T_{j}^{(i)}-\frac{\mathbf{v}_{C} \cdot \mathbf{R}_{j}^{(i)}}{c^{2}}\right) \Delta t=\frac{1}{2}\left[c^{2}\left(T_{j}^{(i)}\right)^{2}-\left(\mathbf{R}_{j}^{(i)}\right)^{2}\right],
$$

where

$$
T_{j}^{(i)}=t_{R j}+b_{C}^{(i)}-t_{C},
$$

and

$$
\mathbf{R}_{j}^{(i)}=\mathbf{r}_{C}^{(i)}+\mathbf{v}_{C} T_{j}^{(i)}-\mathbf{r}_{j} .
$$

This system of equations is of the same form as that discussed in Appendix B and can be solved by iteration in a similar manner as that discussed at the end of Appendix B. The main difference from eq (B.3) is that the velocity estimate $\mathbf{v}_{C}$ appears in eq (C.7) in three places: both on the left and the right sides in the definition of $\mathbf{R}_{j}^{(i)}$, and in the coefficient of $\Delta t$ on the left side.

Thus the iteration scheme proceeds as follows.

Step 1. Choose $t_{C}$; estimate $\mathbf{v}_{C}$ in the ECIF. This will be a combination of velocity relative to the ground, and velocity of the ground relative to the chosen ECIF, due to earth rotation. 
Step 2. Choose approximate values for the user position $\mathbf{r}_{C}$ and clock bias $b_{C}$. Compute $\mathbf{R}_{j}^{(i)}$ and $T_{j}^{(i)}$.

Step 3. Solve eqs (C.7) to obtain corrections $\Delta \mathbf{r}$ and $\Delta t$ for user position and clock bias.

Step 4. Test whether the corrections are sufficiently small that computation can be halted; if not combine the corrections obtained in Step 3 with the approximate values of position and bias, to obtain a new approximate position and bias.

Step 5. Recompute $\mathbf{R}_{j}^{(i)}$ and $T_{j}^{(i)}$ and return to Step 3 . 


\section{Appendix D.}

\section{Equivalence of Integrated Doppler and One-Way Light Time}

In this Appendix we show the complete equivalence of the one-way light time measurement to integrated Doppler measurements.

Neglecting gravitational path bending and gravitational time delay is well justified; electromagnetic signals travel with speed $c$ in straight lines in the ECI reference frame. The direction of propagation may then be described by a constant unit vector $\mathrm{N}$ along the path from transmitter to receiver.

Let the coordinate time of transmission of a signal be denoted by $t_{T}$ and the coordinate time of reception be denoted by $t_{R}$. The positions of the transmission and reception events in ECI coordinates are denoted by $\mathbf{r}_{T}\left(t_{T}\right)$ and $\mathbf{r}_{R}\left(t_{R}\right)$. Also, the velocities of the transmitter and receiver at the transmission and reception epochs are denoted by $\mathbf{V}$ and $\mathbf{v}$, respectively; this notation is introduced in eqs (1-2) where the Doppler effect was stated. In this Appendix we derive the Doppler formula including gravitational frequency shifts.

The one-way propagation of a signal from transmitter to receiver gives

$$
t_{R}=K+t_{T}+\frac{1}{c}\left|\mathbf{r}_{R}\left(t_{R}\right)-\mathbf{r}_{T}\left(t_{T}\right)\right|,
$$

where $\mathrm{K}$ represents a possible systematic error or bias in the coordinate time of the receiver clock. The term $\left|\mathbf{r}_{R}\left(t_{R}\right)-\mathbf{r}_{T}\left(t_{T}\right)\right| / c$ is the propagation delay in the equation, which must be accounted for in any use of GPS signals for navigation or time transfer. We show here that if a receiver properly accounts for this term, then it has automatically accounted for the relativistic Doppler effect, integrated over time.

Let there be a second signal sent from transmitter to receiver after one period of oscillation of the oscillator in the transmitter. Let the times of transmission and reception of this second signal be $t_{T}+d t_{T}$ and $t_{R}+d t_{R}$. The frequencies will be very high, so the small differentials may be calculated by taking differentials of eq (D.1). The term in $K$ drops out, and

$$
d t_{R}=d t_{T}+\frac{1}{c} \frac{\mathbf{r}_{R}\left(t_{R}\right)-\mathbf{r}_{T}\left(t_{T}\right)}{\left|\mathbf{r}_{R}\left(t_{R}\right)-\mathbf{r}_{T}\left(t_{T}\right)\right|} \cdot\left(d \mathbf{r}_{R}-d \mathbf{r}_{T}\right),
$$

where $d \mathbf{r}_{R}$ and $d \mathbf{r}_{T}$ are the changes in positions of the receiver and transmitter during the time intervals $d t_{R}$ and $d t_{T}$. But the unit vector $\mathrm{N}$ is exactly

$$
\mathbf{N}=\frac{\mathbf{r}_{R}\left(t_{R}\right)-\mathbf{r}_{T}\left(t_{T}\right)}{\left|\mathbf{r}_{R}\left(t_{R}\right)-\mathbf{r}_{T}\left(t_{T}\right)\right|}
$$

and during the small time intervals $d t_{R}, d t_{T}$ we have

$$
d \mathbf{r}_{R}=\mathbf{v} d t_{R}, \quad d \mathbf{r}_{T}=\mathbf{V} d t_{T} .
$$


Therefore

$$
d t_{R}=d t_{T}+\frac{1}{c} \mathbf{N} \cdot\left(\mathbf{v} d t_{R}-\mathbf{V} d t_{T}\right)
$$

Rearranging gives

$$
d t_{R}\left(1-\frac{\mathbf{N} \cdot \mathbf{v}}{c}\right)=d t_{T}\left(1-\frac{\mathbf{N} \cdot \mathbf{V}}{c}\right) .
$$

Next we relate the coordinate time intervals $d t_{R}, d t_{T}$ to the proper frequencies of received signal and transmitted signal. This part of the calculation is only carried to terms of order $c^{-2}$. Let $\Phi(\mathbf{r})$ be the Newionian gravitational potential at position $\mathbf{r}$ in ECI coordinates. Let

$$
\Delta \Phi=\Phi-\Phi_{0},
$$

where the constant $\Phi_{0}$ is the effective gravitational potential on the rotating geoid, including the centripetal contribution arising from earth's spin. Then the proper time $d \tau$ that elapses on an atomic clock as it moves through the position increment $d \mathbf{r}$ during the coordinate time increment $d t$ is given approximately according to general relativity by $[2,3]$

$$
c^{2} d \tau^{2}=\left(1+\frac{2 \Delta \Phi}{c^{2}}\right) c^{2} d t^{2}-(d \mathbf{r})^{2}=\left(1+\frac{2 \Delta \Phi}{c^{2}}-\frac{(d \mathbf{r} / d t)^{2}}{c^{2}}\right) c^{2} d t^{2} .
$$

This may be solved for the coordinate time increment $d t$ in terms of the proper time:

$$
d t=\frac{d \tau}{\sqrt{\left(1+2 \Delta \Phi / c^{2}-(d \mathbf{r} / d t)^{2} / c^{2}\right)}} \approx \frac{d \tau}{1+\frac{\Delta \Phi}{c^{2}}-\frac{1}{2} \frac{(d \mathbf{r} / d t)^{2}}{c^{2}}} .
$$

where the square root has been expanded using the first term in a binomial expansion, $\sqrt{1+x / c^{2}} \approx 1+x /\left(2 c^{2}\right)$. This may be applied to the coordinate time intervals $d t_{R}$ and $d t_{T}$ between receptions and transmissions of the two successive signals. Accurate to terms of order $1 / c^{2}$,

$$
\begin{aligned}
d t_{R} & =\frac{d \tau_{R}}{1+\frac{\Delta \Phi\left(\mathbf{r}_{R}\right)}{c^{2}}-\frac{1}{2} \frac{\mathbf{v}^{2}}{c^{2}}} . \\
d t_{T} & =\frac{d \tau_{T}}{1+\frac{\Delta \Phi\left(\mathbf{r}_{T}\right)}{c^{2}}-\frac{1}{2} \frac{\mathbf{V}^{2}}{c^{2}}} .
\end{aligned}
$$

We substitute these results into eq (D.6) and obtain

$$
\frac{d \tau_{R}\left(1-\frac{\mathbf{N} \cdot \mathbf{v}}{c}\right)}{1+\frac{\Delta \Phi\left(\mathbf{r}_{R}\right)}{c^{2}}-\frac{1}{2} \frac{\mathbf{v}^{2}}{c^{2}}}=\frac{d \tau_{T}\left(1-\frac{\mathbf{N} \cdot \mathbf{V}}{c}\right)}{1+\frac{\Delta \Phi\left(\mathbf{r}_{T}\right)}{c^{2}}-\frac{1}{2} \frac{\mathbf{V}^{2}}{c^{2}}} .
$$

The last step is to relate the proper time intervals to the proper frequencies. Using $F$ and $f$ for the proper transmitted and received frequencies, respectively, we find

$$
F=\frac{1}{d \tau_{T}}, \quad f=\frac{1}{d \tau_{R}} .
$$


And substitution into eq (D.12) gives the final relation between proper frequencies:

$$
\frac{f\left(1+\Delta \Phi\left(\mathbf{r}_{R}\right) / c^{2}-\mathbf{v}^{2} / 2 c^{2}\right)}{1-\frac{\mathbf{N} \cdot \mathbf{v}}{c}}=\frac{F\left(1+\Delta \Phi\left(\mathbf{r}_{T}\right) / c^{2}-\mathbf{V}^{2} / 2 c^{2}\right)}{1-\frac{\mathbf{N} \cdot \mathbf{V}}{c}} .
$$

Upon neglecting the gravitational frequency shifts, this form agrees with eq (1) in the main text to the required order of approximation.

Several observations about the result in eq (D.14) are appropriate. First, all quantities, except the proper frequencies, are measured in ECI coordinates. Second, there are no terms of the form $\mathbf{v} \cdot \mathbf{V} / c^{2}$. Third, to obtain the ratio $f / F$ or $F / f$ correct to order $1 / c^{2}$, we must take care to expand the aberration factors $\left(1-\mathrm{N} \cdot \mathbf{v} / c^{2}\right)^{-1}$ and $\left(1-\mathrm{N} \cdot \mathrm{V} / c^{2}\right)^{-1}$ to the required order. To illustrate this last point, let us calculate the ratio $F / f$. Expanding eq (D.14) to second order gives

$$
\begin{aligned}
F / f=1+ & \frac{\Phi\left(\mathbf{r}_{R}\right)-\Phi\left(\mathbf{r}_{T}\right)}{c^{2}}-\frac{\mathbf{v}^{2}}{2 c^{2}}+\frac{\mathbf{V}^{2}}{2 c^{2}} \\
& +\frac{\mathbf{N} \cdot(\mathbf{v}-\mathbf{V})}{c}-\frac{\mathbf{N} \cdot \mathbf{v}}{c} \frac{\mathbf{N} \cdot \mathbf{V}}{c}+\left(\frac{\mathbf{N} \cdot \mathbf{v}}{c}\right)^{2} .
\end{aligned}
$$

Conservation of coordinate frequency. Consider the special case when both the transmitter and the receiver are at rest in the ECI reference frame. Then eq (D.6) gives

$$
d t_{R}=d t_{T}
$$

This means that the coordinate time between reception of successive wavefronts is the same as the coordinate time between the emission of successive wavefronts. The coordinate frequency is the reciprocal of this time, so this result states that for observers at rest in the ECI reference frame, coordinate frequency is conserved. This is a well-known fact that can be proved by separate arguments whenever the metric tensor coefficients do not depend on the time. This is the case here.

We may readily reverse the argument given above and pass from eq (D.14) back to eq (D.1) by integration rather than differentiation. Equation (D.6) is an intermediate step in such an argument, which is obtained by combining eqs (D.10, D.11, and D.12) so as to eliminate the proper time intervals $d \tau_{R}$ and $d \tau_{T}$. Then eq (D.2) follows from (D.3-5). Integration then yields eq (D.1) with an unknown constant of integration.

Thus the change in the one-way light time, as used in GPS for pseudorange, is equal to the integrated Doppler frequency shift over the interval of the change using the frequency relation (D.13). 


\section{Appendix E.}

\section{Numerical Check on the First-Order Doppler Correction, Equation (16)}

In this Appendix we use data provided in Example 5.1 to compare the "true" value of $\Delta t_{R j}$ from the simulation with values obtained using eq (16). For the case of satellite 1 in Example 5.1,

$$
\Delta t_{S V}=-0.0000565547962 \mathrm{~s} .
$$

Then eq (16) gives

$$
\Delta t_{R 1}=-0.000056554784 \mathrm{~s} .
$$

This estimate using first-order Doppler on the coordinate time may be compared with the simulation which gives for the two arrival times of the position in the code train corresponding to $t_{S V}$ and $t_{C}$ the following time difference:

$$
\begin{aligned}
\Delta t_{R 1} & =240.069124140263-240.069180695048 \mathrm{~s} \\
& =-0.000056554785 \mathrm{~s} .
\end{aligned}
$$

The extremely good agreement between these numbers-one calculated using the Doppler formula and one using propagation delay equations - suggests the following strategy for measuring the coordinate time of arrival of the code corresponding to the chosen measurement time $t_{C}$. If we choose a time $t_{C}$ as some multiple of $1 \mathrm{~ms}$ so that it lies on a code boundary, the SV clock correction $\Delta t_{S V j}$ can be computed from the navigation message. The receiver can then measure the arrival time $t_{R j}$ of the code boundary $t_{S V j}$ which is equal numerically to $t_{C}$ (arrival of phase time $T=t_{C}$ ). Then the Doppler correction factor given in eq (16) can be computed by the receiver and added to $t_{R j}$ to predict, with excellent accuracy, the arrival time $t_{R j}+d t_{R j}$ of the code which corresponds to transmission of $t_{C}$ from the satellite. Thus only times of arrival of code boundaries would actually be measured by the receiver.

Three additional calculations based on eq (16) are given in Table E.1, for the four satellites used in Example 5.1. The simulated intervals $\Delta t_{S V j}$ are given in the first column of Table E.1. The second column lists the values of $\Delta t_{R j}$ obtained using eq (16). For comparison, the last column gives the "true" values of $\Delta t_{R j}$ obtained from the simulation. In all cases the first-order Doppler correction, eq (16), agrees extremely well with the "true" values. The corrections have been incorporated into the data listed in Table 7 . 
Table E.1. Computation of first-order Doppler corrections.

$$
\text { (units }=\mathrm{s} \text { ) }
$$

\begin{tabular}{crrr}
\hline ID \# & \multicolumn{1}{c}{$\Delta t_{S V j}$} & \multicolumn{1}{c}{$\Delta t_{R j}$ eq $(16)$} & \multicolumn{1}{c}{$\Delta t_{R j}$} \\
\hline 1 & -0.0000565547962 & -0.000056554784 & -0.000056554785 \\
2 & 0.0004937927006 & 0.000493792194 & 0.000493792194 \\
3 & 0.0002597259299 & 0.000259725401 & 0.000259725400 \\
4 & 0.0003057143767 & 0.000305713988 & 0.000305713988 \\
\hline
\end{tabular}


2 


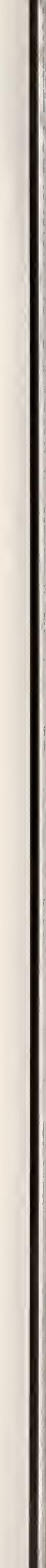




\section{$N I S T_{\text {Technical Pubications }}$}

\section{Periodical}

Journal of Research of the National Institute of Standards and Technology-Reports NIST research and development in those disciplines of the physical and engineering sciences in which the Institute is active. These include physics, chemistry, engineering, mathematics, and computer sciences. Papers cover a broad range of subjects, with major emphasis on measurement methodology and the basic technology underlying standardization. Also included from time to time are survey articles on topics closely related to the Institute's technical and scientific programs. Issued six times a year.

\section{Nonperiodicals}

Monographs-Major contributions to the technical literature on various subjects related to the Institute's scientific and technical activities.

Handbooks-Recommended codes of engineering and industrial practice (including safety codes) developed in cooperation with interested industries, professional organizations, and regulatory bodies.

Special Publications-Include proceedings of conferences sponsored by NIST, NIST annual reports, and other special publications appropriate to this grouping such as wall charts, pocket cards, and bibliographies.

Applied Mathematics Series-Mathematical tables, manuals, and studies of special interest to physicists, engineers, chemists, biologists, mathematicians, computer programmers, and others engaged in scientific and technical work.

National Standard Reference Data Series-Provides quantitative data on the physical and chemical properties of materials, compiled from the world's literature and critically evaluated. Developed under a worldwide program coordinated by NIST under the authority of the National Standard Data Act (Public Law 90-396). NOTE: The Journal of Physical and Chemical Reference Data (JPCRD) is published bimonthly for NIST by the American Chemical Society (ACS) and the American Institute of Physics (AIP). Subscriptions, reprints, and supplements are available from ACS, 1155 Sixteenth St., NW, Washington, DC 20056.

Building Science Series-Disseminates technical information developed at the Institute on building materials, components, systems, and whole structures. The series presents research results, test methods, and performance criteria related to the structural and environmental functions and the durability and safety characteristics of building elements and systems.

Technical Notes-Studies or reports which are complete in themselves but restrictive in their treatment of a subject. Analogous to monographs but not so comprehensive in scope or definitive in treatment of the subject area. Often serve as a vehicle for final reports of work performed at NIST under the sponsorship of other government agencies.

Voluntary Product Standards-Developed under procedures published by the Department of Commerce in Part 10, Title 15, of the Code of Federal Regulations. The standards establish nationally recognized requirements for products, and provide all concerned interests with a basis for common understanding of the characteristics of the products. NIST administers this program in support of the efforts of privatesector standardizing organizations.

Consumer Information Series-Practical information, based on NIST research and experience, covering areas of interest to the consumer. Easily understandable language and illustrations provide useful background knowledge for shopping in today's technological marketplace.

Order the above NIST publications from: Superintendent of Documents, Government Printing Office, Washington, DC 20402.

Order the following NIST publications-FIPS and NISTIRs-from the National Technical Information Service, Springfield, VA 22161.

Federal Information Processing Standards Publications (FIPS PUB)-Publications in this series collectively constitute the Federal Information Processing Standards Register. The Register serves as the official source of information in the Federal Government regarding standards issued by NIST pursuant to the Federal Property and Administrative Services Act of 1949 as amended, Public Law 89-306 (79 Stat. 1127), and as implemented by Executive Order 11717 (38 FR 12315, dated May 11, 1973) and Part 6 of Title 15 CFR (Code of Federal Regulations).

NIST Interagency Reports (NISTIR)-A special series of interim or final reports on work performed by NIST for outside sponsors (both government and non-government). In general, initial distribution is handled by the sponsor; public distribution is by the National Technical Information Service, Springfield, VA 22161, in paper copy or microfiche form. 
U.S. Department of Commerce

National Institute of Standards and Technology

325 Broadway

Boulder, Colorado 80303-3328

Official Business

Penalty for Private Use, $\$ 300$ 